TRANSACTIONS OF THE

AMERICAN MATHEMATICAL SOCIETY

Volume 352, Number 2, Pages 753-780

S 0002-9947(99)02228-X

Article electronically published on October 6, 1999

\title{
OPERATING POINTS IN INFINITE NONLINEAR NETWORKS APPROXIMATED BY FINITE NETWORKS
}

\author{
BRUCE D. CALVERT AND ARMEN H. ZEMANIAN
}

\begin{abstract}
Given a nonlinear infinite resistive network, an operating point can be determined by approximating the network by finite networks obtained by shorting together various infinite sets of nodes, and then taking a limit of the nodal potential functions of the finite networks. Initially, by taking a completion of the node set of the infinite network under a metric given by the resistances, limit points are obtained that represent generalized ends, which we call "terminals," of the infinite network. These terminals can be shorted together to obtain a generalized kind of node, a special case of a 1-node. An operating point will involve Kirchhoff's current law holding at 1-nodes, and so the flow of current into these terminals is studied. We give existence and bounds for an operating point that also has a nodal potential function, which is continuous at the 1-nodes. The existence is derived from the said approximations.
\end{abstract}

\section{INTRODUCTION}

This paper forms part of the body of work that studies existence, uniqueness, bounds, stability, etc. for operating points in nonlinear resistive electrical networks. In particular, we look at infinite networks and how we may naturally and simply consider voltages and currents associated with the ends of the graph or more naturally with certain extremities of the network, which we call "terminals" or more specifically " $s$-terminals", where $s$ may be defined in terms of the resistances or $i-v$ characteristics of the network. We obtain existence results by approximating these infinite networks by finite networks. The latter are formed from subnetworks by identifying various nodes to match the structure of the infinite network. This was stimulated by recent works [1] and [2], in which finite approximations to the infinite network, coupled with a priori bounds that give compactness, lead to the existence of an operating point for the infinite network. This strategy appears in [11].

We note that the investigations of the existence of an operating point involving Kirchhoff's voltage law around infinite loops (i.e., 1-loops), given in [18]-[20], are rather different in that the existence of infinite loop currents is built into the theory through the construction of a solution space [18, pages 77 and 133], [20, page 130].

The relationship of our present result to the approximation theorems in Flanders [6] and Soardi [15] is that in their works there was no shorting at infinity, whereas in

Received by the editors August 5, 1996 and, in revised form, October 17, 1997.

1991 Mathematics Subject Classification. Primary 31C20, 94C05.

Key words and phrases. Infinite resistive networks, ends, metrizing infinite networks, monotone networks, 1-nodes, Kirchhoff's voltage law, Kirchhoff's current law.

This work was partially supported by the National Science Foundation under Grants DMS9200738 and MIP-9423732. 
this paper it is a central issue. In fact, most studies - linear or nonlinear - do not involve Kirchhoff's current law at infinite extremities; see [4], [16], and [17] for such theories. Also, the existence results in [4], [6], [16], [18] and [20] are given directly in infinite dimensions, in contrast to this paper. However, this paper extends some of the work on finitely structured 1-graphs in [20]; the latter does achieve Kirchhoff's current law at cuts around certain transfinite extremities [20, Section 6.2].

We divide this paper into four sections. Its crux is the Section 4 on existence and bounds. But, equally significant is the general study of terminals in Section 2, followed by the study of current flows into terminals in Section 3 .

We conclude this Introduction by going over some terminology. Following Iri [12], to form a directed graph we take a set $\mathcal{B}$ of branches (synonymously, edges), a set $\mathcal{N}^{0}$ of nodes (synonymously, vertices), and an incidence relation consisting of two functions $\partial^{+}: \mathcal{B} \rightsquigarrow \mathcal{N}^{0}$ and $\partial^{-}: \mathcal{B} \rightsquigarrow \mathcal{N}^{0}$. We say that branch $b$ is incident to the node $n$ if $\partial^{+}(b)=n$ or $\partial^{-}(b)=n$.

In this paper there is nothing necessarily or specifically electrical; indeed, Iri [12] explains how transportation problems and project-scheduling problems have the same form. Nonetheless, we will speak in terms of the electrical paradigm. The simplest electrical networks are modeled by a finite digraph together with, for each branch $b$, a set $M_{b} \subset \mathbf{R}^{2}$, which gives the set of possible pairs $\left(i_{b}, v_{b}\right)$ that measure the time-invariant current and voltage in branch $b$. An operating point consists of $\left(i_{b}, v_{b}\right) \in M_{b}$ for each $b$ with $i=\left\{i_{b}\right\}_{b \in \mathcal{B}}$ satisfying Kirchhoff's current law and $v=\left\{v_{b}\right\}_{b \in \mathcal{B}}$ satisfying Kirchhoff's voltage law; these laws are specified below. We may call the network resistive to emphasize that we are not looking at time-dependent behavior.

In this work we not only start with a conventionally infinite network $\left(\mathcal{B}, \mathcal{N}^{0}\right)$, but we then construct generalized nodes, called 1-nodes, at the network's infinite extremities and denote the result by $\left(\mathcal{B}, \mathcal{N}^{0}, \mathcal{N}^{1}\right)$; consequently, the definition of an operating point requires some preliminaries. We say that $\left(\mathcal{B}, \mathcal{N}^{0}\right)$ is locally finite to mean that for every node there are only finitely many branches incident to it; later on we will extend the idea of local finiteness to the 1 -nodes. We say that $i: \mathcal{B} \rightsquigarrow \mathbf{R}^{1}$ satisfies Kirchhoff's current law, when the digraph $\left(\mathcal{B}, \mathcal{N}^{0}\right)$ is locally finite, to mean that its boundary $\partial i: \mathcal{N}^{0} \rightsquigarrow \mathbf{R}^{1}$ is 0 , where for each $n$

$$
\partial i(n)=\sum_{\partial^{+} b=n} i_{b}-\sum_{\partial^{-} b=n} i_{b} .
$$

We say that $v: \mathcal{B} \rightsquigarrow \mathbf{R}^{1}$ satisfies Kirchhoff's voltage law on the digraph $\left(\mathcal{B}, \mathcal{N}^{0}\right)$ if $v$ is a coboundary $\partial p$; that is, there is a function $p: \mathcal{N}^{0} \rightsquigarrow \mathbf{R}^{1}$, called the potential for $v$, with

$$
v_{b}=p\left(\partial^{+} b\right)-p\left(\partial^{-} b\right)
$$

for all $b$. These laws too will be extended to the network augmented by 1-nodes.

As usual, we may consider the digraph $\left(\mathcal{B}, \mathcal{N}^{0}\right)$ in situations that concern only the underlying graph, wherein the branch orientations are ignored; in such cases we may refer to $\left(\mathcal{B}, \mathcal{N}^{0}\right)$ as a graph. If $\mathcal{E} \subset \mathcal{B},\langle\mathcal{E}\rangle$ denotes the digraph induced by $\mathcal{E}$. We say, given $a \in \mathcal{B}$ and $b \in \mathcal{B}$, that $a$ is adjacent to $b$ or that $a$ and $b$ are adjacent to mean that $a \neq b$ and $a$ and $b$ are incident to the same node. Furthermore, that $b$ is adjacent to $\mathcal{E} \subset \mathcal{B}$ means that $b$ is adjacent to some $a \in \mathcal{E}$ and $b \notin \mathcal{E}$. We write $A(\mathcal{E})$ for the set of branches adjacent to $\mathcal{E}$. We say that two nodes $x$ and $y$ are neighbors or are adjacent when there is a branch incident to both $x$ and $y$ and 
$x \neq y$. If $\mathcal{V} \subset \mathcal{N}^{0},\langle V\rangle$ denotes the digraph induced by $\mathcal{V}$. If $\mathcal{V}$ and $\mathcal{W}$ are disjoint subsets of $\mathcal{N}^{0},\langle\mathcal{V}, \mathcal{W}\rangle$ denotes the set of branches incident to a node in $\mathcal{V}$ and a node in $\mathcal{W}$. Given a subgraph $G$ of $\left(\mathcal{B}, \mathcal{N}^{0}\right)$, write $V(G)$ for its node set and $E(G)$ for its branch set.

We use $\mathbb{N}$ for the set $\{0,1,2, \ldots\}$ of natural numbers and $\mathbb{Z}$ for the set $\{\ldots,-1,0,1, \ldots\}$ of integers. A one-ended path may be conceived of equally as:

(i) A function $P$ from $\mathbb{N}$ to $\mathcal{B}$, or from $-\mathbb{N}$ to $\mathcal{B}$, such that $P_{i}$ is adjacent to $P_{j}$ if and only if $|i-j|=1$, and $P_{0}$ is not a self loop.

(ii) A subgraph induced by the range of $P$ as in (i).

(We cannot describe $P$ as a sequence $\left\{x_{i}\right\}$ of distinct nodes with $x_{i}$ and $x_{i+1}$ adjacent because parallel branches are allowed.) We write $\mathcal{O}$ for the set of oneended paths. An endless path $P$ is similarly a function from $\mathbb{Z}$ to $\mathcal{B}$ with $P_{i}$ adjacent to $P_{j}$ if and only if $|i-j|=1$ or equally is a subgraph induced by the range of such a function.

We recall definitions and notations concerning monotone networks in Section 4, and generally recall definitions as they are used.

We use the symbols $\vee$ and $\wedge$ to denote max and min respectively. We write $(\widehat{\mathcal{X}}, d)$ for the completion of the metric space $(\mathcal{X}, d)$. We use $\mathcal{A}^{\prime}$ to denote the set of limit points (or, synonymously, cluster points) of $\mathcal{A}$, a subset of a given topological space. We write $\mathcal{U}(x)$ for the set of open neighborhoods of the element $x$ of a given topological space. We use $\mathcal{U}^{*}(x)$ for the set of neighborhoods $M$ of $x$ that are both open and closed and for which $\left\langle M \cap \mathcal{N}^{0}\right\rangle$ is a connected graph, where now $x$ is a point in a topological space containing $\mathcal{N}^{0}$.

\section{2. $s$-TERMINALS AND ENDS}

Rather than starting with an infinite graph and determining its infinite extremities, that is, its "ends" — as is done in [3], [7], [8], [9], [14], [3], [16], and [17], we start with an infinite electrical network and devise a metric on the network's nodes based on the $(i, v)$-characteristics of the branches. We then determine infinite extremities, which we call "terminals" or more specifically " $R$-terminals," by completing the network under that metric. More generally, we construct "s-terminals" in the next definition. Our $s$-terminals encompass, as special cases, the strictly graphtheoretical ends of Freudenthal and Halin; $s$-terminals expand into ends when $s$ is summable.

Definition 2.1. Let $\left(\mathcal{B}, \mathcal{N}^{0}\right)$ be a locally finite graph, and let $s: \mathcal{B} \rightsquigarrow(0, \infty)$ be given. Define the metric $d_{s}$ on $V(\mathcal{G})$ for each component $\mathcal{G}$ of $\left(\mathcal{B}, \mathcal{N}^{0}\right)$ by $d_{s}(x, x)=0$ and

$$
d_{s}(x, y)=\inf \left\{\sum_{b \in E(P)} s_{b}: P \text { a path connecting } x \text { and } y\right\} .
$$

Write $\left(\widehat{V(\mathcal{G})}, d_{s}\right)$ for the completion, and call any limit point or cluster point (i.e., any element of $\left.\widehat{V(\mathcal{G}}^{\prime}\right)$ an $s$-terminal of $\left(\mathcal{B}, \mathcal{N}^{0}\right)$ and say it is in $\mathcal{G}$ when we wish to specify $\mathcal{G}$. We often refer to an $s$-terminal simply as a terminal when the function $s$ is understood.

Remark. Since $\left(\mathcal{B}, \mathcal{N}^{0}\right)$ is locally finite, $d_{s}$ is a metric; in particular, $x \neq y \Rightarrow$ $d_{s}(x, y)>0$. Also, note that Definition 2.14 extends this definition. 


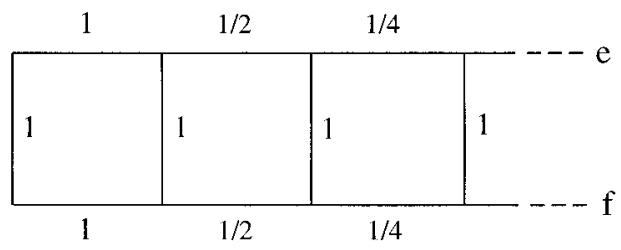

Figure 1. The illustration for Example 2.2. A ladder network without sources. The numbers designate branch resistances. With $s_{b}$ being the resistance of branch $b, e$ and $f$ are $s$-terminals.

Remark. In Section 4 we will choose $s: \mathcal{B} \rightsquigarrow(0, \infty)$ as follows. When $b$ is a branch in Norton form with linear resistance $r_{b} \geq 0$ and current source $h_{b} \in \mathbb{R}^{1}$, we set $s_{b}=r_{b}+t_{b}$, where $t_{b}>0$ is a term to ensure $s_{b}>0$ with $\sum_{b \in \mathcal{B}} t_{b}<\infty$. In the monotone nonlinear case we take $s_{b}=R_{b}^{*}$, where $R_{b}^{*}$ is defined in Section 4 .

Example 2.2. For the ladder graph of Figure 1, if $s_{b}=r_{b}$, there are exactly two $s$-terminals $e$ and $f$. In this case, there is an isometry of $\left(\mathcal{N}^{0}, d_{s}\right)$ into $\mathbb{R}^{2}$, providing thereby a visualization of $\left(\widehat{\mathcal{N}^{0}}, d_{s}\right)$ and hence of the $s$-terminals.

Theorem 2.3. Let $\left(\mathcal{B}, \mathcal{N}^{0}\right)$ be a connected locally finite graph, and let $s: \mathcal{B} \rightsquigarrow$ $(0, \infty)$ be given. Then, for any s-terminal $f$ and one-ended path $P$ with node sequence $\left(y_{0}, y_{1}, \ldots\right)$ such that $y_{n} \rightarrow f$, we have

$$
d_{s}\left(y_{0}, f\right) \leq \sum_{b \in E(P)} s_{b}
$$

Moreover, for any $v \in \mathcal{N}^{0}$, any s-terminal $f$, and any $\epsilon>0$, there exists a oneended path $P$ with node sequence $\left(y_{0}, y_{1}, \ldots\right)$ such that $y_{0}=v, y_{n} \rightarrow f$, and

$$
\sum_{b \in E(P)} s_{b}<d_{s}\left(y_{0}, f\right)+\epsilon .
$$

Proof. Let $\left(b_{1}, b_{2}, \ldots\right)$ be the branch sequence of $P$. For any $n \geq 1, d_{s}\left(y_{0}, y_{n}\right) \leq$ $\sum_{i=1}^{n} s_{b_{i}} \leq \sum_{b \in E(P)} s_{b}$. Letting $n \rightarrow \infty$, we obtain (1).

To show the existence of $P$, we start by taking $x_{n} \in \mathcal{N}^{0}$ for $n \geq 1$ with $d_{s}\left(x_{n}, f\right)<\epsilon /\left(2^{n} 3\right)$. Take $P_{0}$ as a path connecting $v$ and $x_{1}$ with $\sum_{b \in E\left(P_{0}\right)} s_{b}<$ $d_{s}(v, f)+\epsilon / 2$. For $n=1,2, \ldots$ take $P_{n}$ as a path connecting $x_{n}$ and $x_{n+1}$ with $\sum_{b \in E\left(P_{n}\right)} s_{b}<\epsilon / 2^{n+1}$, which can be done because $d_{s}\left(x_{n}, x_{n+1}\right) \leq d_{s}\left(x_{n}, f\right)+$ $d_{s}\left(x_{n+1}, f\right)<\epsilon / 2^{n+1}$. Let $Q$ be the infinite, locally finite graph $\bigcup_{n=0}^{\infty} P_{n}$. By Konig's lemma [10, page 81], we can take $P$ to be a one-ended path in $Q$ with initial node $v$. Now,

$$
\begin{aligned}
& \sum_{b \in E(P)} s_{b} \leq \sum_{b \in E(Q)} s_{b} \leq \sum_{n} \sum_{b \in E\left(P_{n}\right)} s_{b} \\
& <d_{s}(v, f)+\sum_{n=0}^{\infty} \epsilon / 2^{n+1}=d_{s}(v, f)+\epsilon,
\end{aligned}
$$

whence (2).

Now, if $y \in V\left(P_{n}\right)$ for $n \geq 1$, then

$$
d_{s}(y, f) \leq d_{s}\left(y, x_{n+1}\right)+d_{s}\left(x_{n+1}, f\right)<\epsilon / 2^{n+1}+\epsilon /\left(2^{n+1} 3\right)=\epsilon /\left(2^{n-1} 3\right) .
$$


Let $\left(y_{0}, y_{1}, \ldots\right)$ be the node sequence of $P$. Since the $P_{i}$ are all finite paths, given any $N,\left(y_{1}, y_{2}, \ldots\right)$ is eventually in $\bigcup_{k \geq N} V\left(P_{k}\right)$, and so for large enough $n, d_{s}\left(y_{n}, f\right)<\epsilon /\left(2^{N-1} 3\right)$. Hence, $y_{n} \rightarrow f$.

Corollary 2.4. Let $\left(\mathcal{B}, \mathcal{N}^{0}\right)$ and $s$ be as in Theorem 2.3. The $s$-terminals bijectively correspond with $R$-equivalence classes of the set $\mathcal{O}_{s}$ of one-ended paths $P$ with $\sum_{b \in E(P)} s_{b}<\infty$, where $R$ is defined by $P^{1} R P^{2}$ if and only if for every $K>0$ there are infinitely many paths $Q$ connecting a node of $P^{1}$ and a node of $P^{2}$ with $\sum_{b \in E(Q)} s_{b}<K$. The correspondence maps the equivalence class RP to the limit of the node sequence of $P$, while to the s-terminal e there corresponds the set of paths $P$ in $\mathcal{O}_{s}$ whose node sequences converge to $e$.

Proof. One checks that $R$ is a transitive relation on $\mathcal{O}_{s}$ and hence an equivalence relation. We may define $\phi: \mathcal{O}_{s} \rightsquigarrow \widehat{\mathcal{N}}^{\prime}$ taking $P$ to the limit of its node sequence, since this is a Cauchy sequence for $P \in \mathcal{O}_{s}$. We want to show that $\phi$ is constant on equivalence classes under $R$. Let $P^{1} R P^{2}$, where $P^{1}$ has the node sequence $\left(x_{0}^{1}, x_{1}^{1}, \ldots\right)$ and $P^{2}$ has the node sequence $\left(x_{0}^{2}, x_{1}^{2}, \ldots\right)$. Given any $\epsilon>0$, there is an $N$ such that, for all $j \geq N, d_{s}\left(x_{j}^{1}, \phi\left(P^{1}\right)\right)<\epsilon / 3, d_{s}\left(x_{j}^{2}, \phi\left(P^{2}\right)\right)<\epsilon / 3$, and moreover there is a path $Q$ connecting a node $x_{n}^{1}$ of $P^{1}$ and a node $x_{m}^{2}$ of $P^{2}$ such that $m \geq N, n \geq N$, and $\sum_{b \in E(Q)} s_{b}<\epsilon / 3$, the third fact being a consequence of local finiteness. Thus, $d_{s}\left(\phi\left(P^{1}\right), \phi\left(P^{2}\right)\right)<\epsilon$, and consequently, $\phi\left(P^{1}\right)=\phi\left(P^{2}\right)$.

Furthermore, $\phi$ induces a map, also denoted by $\phi$, on $\mathcal{O}_{s} / R$. By Theorem 2.3, $\phi$ maps onto $\widehat{\mathcal{N}}^{\prime}$. To see that $\phi$ is one-to-one on $\mathcal{O}_{s} / R$, note that, if $P^{1}$ and $P^{2}$ are not equivalent, there is a $K>0$ and only finitely many pairs of nodes $x^{1}$ from $P^{1}$ and $x^{2}$ from $P^{2}$ with $d_{s}\left(x^{1}, x^{2}\right)<K$. Since, for $j=1,2, x_{n}^{j} \rightarrow \phi\left(P^{j}\right)$ as $n \rightarrow \infty$, $d_{s}\left(\phi\left(P^{1}\right), \phi\left(P^{2}\right)\right) \geq K$, and therefore $\phi\left(P^{1}\right) \neq \phi\left(P^{2}\right)$.

Terminology. We may refer to these equivalence classes of $\mathcal{O}_{s}$ as $s$-terminals, and say " $P$ is in the $s$-terminal $f$ " when $P \in \mathcal{O}_{s}$ has the node sequence $\left(y_{0}, y_{1}, \ldots\right)$ with $y_{n} \rightarrow f$. We may also say " $P$ is a one-ended path connecting $y_{0}$ and $f . "$

Corollary 2.5. Let $\left(\mathcal{B}, \mathcal{N}^{0}\right)$ be connected and locally finite, and let $s: \mathcal{B} \rightsquigarrow(0, \infty)$ be given. Given $f$ an s-terminal and $\epsilon>0$, the ball $B(f, \epsilon)$ in $\left(\widehat{\mathcal{N}^{0}}, d_{s}\right)$ has the property that $\left\langle B(f, \epsilon) \cap \mathcal{N}^{0}\right\rangle$ is connected.

Proof. By Theorem 2.3, given any two nodes in $B(f, \epsilon) \cap \mathcal{N}^{0}$, there are two oneended paths connecting those nodes to $f$. Then, by Corollary 2.4, there is at least one path connecting a node of one path to a node of the other path that remains within $B(f, \epsilon)$.

Corollary 2.6. Let $G=\left(\mathcal{B}, \mathcal{N}^{0}\right)$ be connected and locally finite, and let $s: \mathcal{B} \rightsquigarrow$ $(0, \infty)$ be given. Given two s-terminals $e$ and $f$ in $G$, there is "an endless path $P$ connecting e and $f$," (i.e., there is a function $P: \mathbb{Z} \rightsquigarrow \mathcal{B}$ with, for all $m, n, P_{n}$ is adjacent to $P_{m}$ if and only if $|m-n|=1$ and with $\left.P\right|_{\mathbb{N}}$ in $f$ and $\left.P\right|_{(-\mathbb{N})}$ in e), and, given $\epsilon>0$, we may take $P$ with $\sum_{b \in P} s_{b}<d_{s}(e, f)+\epsilon$.

Proof. Let $P^{f} \in \mathcal{O}_{s}$ with node sequence $y_{n} \rightarrow f$, and let $P^{e}$ be in the $s$-terminal $e \neq f$. We see that, for large $n, y_{n}$ is not a node in $P^{e}$, otherwise there would be a subsequence converging to $e$. Suppose $P^{e}$ connects $y_{0}$ to $e$. Let $N=$ $\max \left\{n: y_{n} \in V P^{e}\right\}$ since this is nonempty and bounded above. Combining the 
paths connecting $y_{N}$ to $e$ in $P^{e}$ and $y_{N}$ to $f$ in $P^{f}$ gives $P$. That $P^{e}$ and $P^{f}$ can be chosen so that $P$ satisfies the inequality can be seen as follows.

We can choose a node $x$ such that $d_{s}(x, e)<\epsilon / 2$. By Theorem 2.3, there is a path $Q$ connecting $x$ and $e$ such that $\sum_{b \in E(Q)} s_{b}<\epsilon / 2$. Moreover, $d_{s}(x, f) \leq$ $d_{s}(x, e)+d_{s}(e, f)<\epsilon / 2+d_{s}(e, f)$. Again, by Theorem 2.3, there is a path $L$ connecting $x$ and $f$ such that $\sum_{b \in E(L)} s_{b}<d_{s}(e, f)+\epsilon / 2$. Now, $Q \cup L$ contains an endless path $P$ connecting $e$ and $f$, and moreover

$$
\begin{aligned}
\sum_{b \in E(P)} s_{b} & \leq \sum_{b \in E(Q \cup L)} s_{b} \leq \sum_{b \in E(Q)} s_{b}+\sum_{b \in E(L)} s_{b} \\
& <\epsilon / 2+d_{s}(e, f)+\epsilon / 2 .
\end{aligned}
$$

Now, always assuming $\left(\mathcal{B}, \mathcal{N}^{0}\right)$ to be locally finite, we check the relationship of $s$-terminals to ends, but first recalling their definition and some results, since ends can be thought of in several ways [7], [8], [16], [17].

Definition 2.7. Let $\left(\mathcal{B}, \mathcal{N}^{0}\right)$ be a locally finite graph. An end in $\left(\mathcal{B}, \mathcal{N}^{0}\right)$ is a function $f$ taking each finite subset $\mathcal{F}$ of $\mathcal{B}$ to an infinite component of $\left(\mathcal{B} \backslash \mathcal{F}, \mathcal{N}^{0}\right)$ such that, if $\mathcal{E} \subset \mathcal{F}$, then $f(\mathcal{F}) \subset f(\mathcal{E})$. Write $\partial\left(\mathcal{B}, \mathcal{N}^{0}\right)$ for the set of ends.

Definition 2.8. With $\left(\mathcal{B}, \mathcal{N}^{0}\right)$ being a locally finite graph and $\mathcal{O}$ its set of oneended paths, define the equivalence relation $R$ on $\mathcal{O}$ by $P^{1} R P^{2}$ if and only if, for every finite $\mathcal{E} \subset \mathcal{B}$, there is a component $C$ of $\left(\mathcal{B} \backslash \mathcal{E}, \mathcal{N}^{0}\right)$ with $P_{n}^{1} \in E(C)$ and $P_{n}^{2} \in E(C)$ for all large $n$.

The next result follows from Theorem 1 of Cartwright, Soardi and Woess [3], but we give here a more elementary proof of it. This gives an extension of Konig's lemma, which is further extended by Theorem 2.3 in conjunction with Theorem 2.12 .

Theorem 2.9. Suppose $\left(\mathcal{B}, \mathcal{N}^{0}\right)$ is a locally finite graph. Then, the set of ends in $\left(\mathcal{B}, \mathcal{N}^{0}\right)$ bijectively correspond with the set of equivalence classes of $\mathcal{O}$ under $R$, that correspondence being given by

$$
f \mapsto\left\{P \in \mathcal{O}: \text { for all finite } \mathcal{F} \subset \mathcal{B}, P_{n} \in E(f(\mathcal{F})) \text { for all large } n\right\} \text {. }
$$

Proof. Given the finite set $\mathcal{F} \subset \mathcal{B}$, each one-ended path can meet $\langle\mathcal{F}\rangle$ only finitely many times and therefore must eventually be confined to an infinite component of $\mathcal{B} \backslash \mathcal{F}$. This gives a function $\phi: \mathcal{O} \rightsquigarrow \partial\left(\mathcal{B}, \mathcal{N}^{0}\right)$ taking any one-ended path $P$ to the end $\phi(P)$ defined by $\phi(P)(\mathcal{F})$ equal to the infinite component of $\left(\mathcal{B} \backslash \mathcal{F}, \mathcal{N}^{0}\right)$ that $P$ is eventually in. We note that $\phi(P)$ is indeed an end; i.e., if $\mathcal{E} \subset \mathcal{F}$, then $\phi(P)(\mathcal{E}) \supset \phi(P)(\mathcal{F})$. Since $\phi$ is constant on equivalence classes under $R$, it induces a function, also denoted by $\phi$, from $\mathcal{O} / R$ to $\partial\left(\mathcal{B}, \mathcal{N}^{0}\right)$. Note that $\phi$ is one-to-one. We claim that $\phi$ is surjective. This will complete the proof since $\phi$ gives the inverse of the correspondence described in the theorem.

To proceed, we want to show that given an end $f$ as in Definition 2.7 there is a $P \in \mathcal{O}$ such that, for all finite $\mathcal{E} \subset \mathcal{B}, P$ is eventually confined to $f(\mathcal{E})$. Choose any node $a_{0}$, and let $\mathcal{E}_{1}$ be the set of branches incident to $a_{0}$. Take a sequence $\left\{\mathcal{E}_{n}\right\}_{n=1}^{\infty}$ of branch sets with $\mathcal{E}_{n+1} \supset\left(A\left(\mathcal{E}_{n}\right) \cup \mathcal{E}_{n}\right)$ for each $n$. Take $a_{1} \in V\left(f\left(\mathcal{E}_{1}\right)\right) \cap V\left\langle\mathcal{E}_{1}\right\rangle$ and $P^{0}$ the (one branch) path in $\left\langle\mathcal{E}_{1}\right\rangle$ connecting $a_{0}$ and $a_{1}$. For $n=1,2, \ldots$, given $a_{n} \in V f\left(\mathcal{E}_{n}\right) \cap V\left\langle\mathcal{E}_{n}\right\rangle$, we claim that there is a path $P^{n}$ in $f\left(\mathcal{E}_{n}\right) \backslash f\left(\mathcal{E}_{n+1}\right)$ connecting $a_{n}$ to $a_{n+1} \in V f\left(\mathcal{E}_{n+1}\right) \cap V\left\langle\mathcal{E}_{n+1}\right\rangle$. Let $v \in V f\left(\mathcal{E}_{n+1}\right)$, and take $R$ as a path in $f\left(\mathcal{E}_{n}\right)$ connecting $a_{n}$ and $v$ with initial node $a_{n}$. Then, take $a_{n+1}$ as the 
first node in $V f\left(\mathcal{E}_{n+1}\right)$ that $R$ meets. This gives $P^{n}$ as the part of $R$ connecting $a_{n}$ and $a_{n+1}$, proving the claim.

Finally, set $P=\bigcup_{n=0}^{\infty} P^{n} ; P$ is a one-ended path starting at $a_{0}$. Given any finite branch set $\mathcal{E}$, we may take $\mathcal{E}_{n}$ with $\mathcal{E}_{n} \supset \mathcal{E}$, giving $P$ eventually in $f\left(\mathcal{E}_{n}\right) \subset f(\mathcal{E})$.

As a result of Theorem 2.9, the equivalence classes of Definition 2.8 are also defined as ends, and we shall say that a one-ended path $P$ is in an end $e$. We give a metric $d_{\mathcal{E}}$ related to the sets above.

Proposition 2.10. Let $G=\left(\mathcal{B}, \mathcal{N}^{0}\right)$ be a connected locally finite graph. Suppose $\mathcal{E}=\left\{\mathcal{E}_{n}\right\}_{n=1}^{\infty}$ is a sequence of finite branch sets with $\mathcal{E}_{1}=\emptyset, \bigcup_{n=1}^{\infty} \mathcal{E}_{n}=\mathcal{B}$, and $\mathcal{E}_{n}$ increasing with $n$. For $u, v \in V(G)$ and $e, f \in \partial G$, if

$$
\begin{gathered}
d_{\mathcal{E}}(u, f)=\inf \left\{n^{-1}: u \in V f\left(\mathcal{E}_{n}\right)\right\}, \\
d_{\mathcal{E}}(e, f)=\inf \left\{n^{-1}: e\left(\mathcal{E}_{n}\right)=f\left(\mathcal{E}_{n}\right)\right\},
\end{gathered}
$$

and

$$
d_{\mathcal{E}}(u, v)=\inf \left\{n^{-1}: \text { u and } v \text { connected in }\left(\mathcal{B} \backslash \mathcal{E}_{n}, \mathcal{N}^{0}\right)\right\},
$$

then $d_{\mathcal{E}}$ is a metric on $V(G) \cup \partial G$.

Proof. We see that $d_{\mathcal{E}}(u, u)=0$ since $u$ is connected to itself in all $\left(\mathcal{B} \backslash \mathcal{E}_{n}, \mathcal{N}^{0}\right)$. For the triangle inequality, we claim for $u, v, w \in \mathcal{N}^{0}$ that

$$
d_{\mathcal{E}}(u, v) \leq d_{\mathcal{E}}(u, w) \vee d_{\mathcal{E}}(w, v) .
$$

Suppose the right-hand side is equal to $1 / N$. Then, $u$ and $w$ are connected in $\left(\mathcal{B} \backslash \mathcal{E}_{N}, \mathcal{N}^{0}\right)$, and so too are $w$ and $v$, giving $u$ and $v$ are connected also in $\left(\mathcal{B} \backslash \mathcal{E}_{N}, \mathcal{N}^{0}\right)$ and thus $d_{\mathcal{E}}(u, v) \leq 1 / N$. Hence, $d_{\mathcal{E}}(u, v) \leq d_{\mathcal{E}}(u, w)+d_{\mathcal{E}}(w, v)$. The same argument holds for any combination of nodes and ends.

We will be interested in elements of $\partial G$ that are isolated, i.e., not in the set $(\partial G)^{\prime}$ of limit points of $\partial G$.

Corollary 2.11. Let $\left(\mathcal{B}, \mathcal{N}^{0}\right)$ be locally finite and connected. Let $e$ be an end. It is isolated if and only if there is a finite set $\mathcal{F} \subset \mathcal{B}$ such that, for all $f, e \neq f \Rightarrow$ $e(\mathcal{F}) \neq f(\mathcal{F})$.

Proof. Only if: Assume $e$ is an isolated end, and let $\mathcal{E}=\left\{\mathcal{E}_{n}\right\}_{n=1}^{\infty}$ be as in Proposition 2.10. Then, there is an $N<\infty$ such that $d_{\mathcal{E}}(e, f)>1 / N$ for every end $f \neq e$. Therefore, $e$ and $f$ select different infinite components of $\mathcal{B} \backslash \mathcal{E}_{N}$. That is, $e\left(\mathcal{E}_{N}\right) \neq f\left(\mathcal{E}_{N}\right)$.

If: Suppose $\mathcal{F} \subset \mathcal{B}$ is finite and, for all ends $f, e \neq f \Rightarrow e(\mathcal{F}) \neq f(\mathcal{F})$. Take $\mathcal{E}_{N} \supset \mathcal{F}$. Then, $e \neq f \Rightarrow e\left(\mathcal{E}_{N}\right) \neq f\left(\mathcal{E}_{N}\right)$, and so $d_{\mathcal{E}}(e, f) \geq 1 / N$ for all $f \neq e$. Therefore, $e$ is isolated.

As the next consideration, we now show that ends and $s$-terminals are the same thing when $s$ is summable. Hence, there are metrics $d_{s}$ on $\mathcal{N}^{0} \cup \partial G$ for all summable $s$, and all these metrics give the same topology as that of any $d_{\mathcal{E}}$.

Theorem 2.12. Let $\left(\mathcal{B}, \mathcal{N}^{0}\right)$ be a connected locally finite graph with $s: \mathcal{B} \rightsquigarrow(0, \infty)$ summable: $\sum_{b \in \mathcal{B}} s_{b}<\infty$. Then, the set of ends (i.e., the equivalence classes of $\mathcal{O}$ ) is the set of $s$-terminals (i.e., the equivalence classes of $\mathcal{O}_{s}$ ). 
Proof. Note that $\mathcal{O}=\mathcal{O}_{s}$ because $\sum_{b \in \mathcal{B}} s_{b}<\infty$; that is, every one-ended path $P$ has $\sum_{b \in E(P)} s_{b}<\infty$. Let $P^{1}$ and $P^{2}$ be in the same $s$-terminal $f$ with node sequences $\left\{x_{n}^{1}\right\}_{n=1}^{\infty}$ and $\left\{x_{n}^{2}\right\}_{n=1}^{\infty}$ both converging to $f$. Since $\inf \left\{s_{b}: b \in \mathcal{F}\right\}>0$ for $\mathcal{F}$ any finite branch set and since $d_{s}\left(x_{n}^{1}, x_{n}^{2}\right) \rightarrow 0, x_{n}^{1}$ and $x_{n}^{2}$ are connected in $\left(\mathcal{B} \backslash \mathcal{F}, \mathcal{N}^{0}\right)$ for $n$ large. Hence, if $P^{1}$ is in the end $e$, so too is $P^{2}$.

Conversely, let the one-ended paths $P^{1}$ and $P^{2}$ be in $\mathcal{O}_{s}$ and in the same end. By Halin [8] there is a one-ended path $Q$ meeting both $P^{1}$ and $P^{2}$ infinitely often. Since $Q \in \mathcal{O}_{s}, P^{1}$ and $Q$ are in the same $s$-terminal, as are $P^{2}$ and $Q$, and so $P^{1}$ and $P^{2}$ are in the same $s$-terminal.

Thus, the equivalence relations $R$ of Corollary 2.4 and Definition 2.8 are the same, and the ends and the $s$-terminals coincide.

Next, we find that, if two functions mapping $\mathcal{B}$ into $(0, \infty)$ differ by a summable function, they give the same $s$-terminals. Similarly, if $K$ is greater than 0 and if two functions differ only where they are greater than $K$, they give the same $s$-terminals.

Proposition 2.13. Let $\left(\mathcal{B}, \mathcal{N}^{0}\right)$ be a connected locally finite graph. Let $\mathcal{S}$ denote the set of all mappings of $\mathcal{B}$ into $(0, \infty)$. Define the equivalence relation $L_{1}$ on $\mathcal{S}$ by $s L_{1} r$ if and only if $\sum_{b \in \mathcal{B}}\left|s_{b}-r_{b}\right|<\infty$. Define the equivalence relation $L_{2}$ on $\mathcal{S}$ by $s L_{2} r$ if and only if there is a $K>0$ such that, for all $b \in \mathcal{B}, s_{b}=r_{b}$ or $s_{b} \wedge r_{b} \geq K$. Define the equivalence relation $L_{3}$ on $\mathcal{S}$ by $s L_{3} r$ if and only if there is a finite sequence $\left\{s=s^{1}, s^{2}, \ldots, s^{n}=r\right\}$ such that, for $i=1, \ldots, n, s_{i-1} L_{1} s_{i}$ or $s_{i-1} L_{2} s_{i}$. Then, $s \mapsto\left(\widehat{\mathcal{N}^{0}}, d_{s}\right)^{\prime}$ is constant on equivalence classes under $L_{3}$.

Proof. Suppose $r \in \mathcal{S}, s \in \mathcal{S}$, and $r=s+t$, where $\sum_{b \in \mathcal{B}}\left|t_{b}\right|<\infty$. Suppose $\left\{x_{n}\right\}$ is a Cauchy sequence in $\left(\mathcal{N}^{0}, d_{s}\right)$. Given $\epsilon>0$, take $\mathcal{E} \subset \mathcal{B}$, finite and with $\sum_{b \in \mathcal{B} \backslash \mathcal{E}}\left|t_{b}\right|<\epsilon / 2$. Take $N_{1}$ and an end $e$ such that $x_{n} \in V e(\mathcal{E})$ if $n \geq N_{1}$, and take $N_{2}$ such that $d_{s}\left(x_{m}, x_{n}\right)<\epsilon / 2$ if $m, n \geq N_{2}$. Also, let $N=N_{1} \vee N_{2}$. For $m, n \geq N$, $d_{r}\left(x_{m}, x_{n}\right)<d_{s}\left(x_{m}, x_{n}\right)+\sum_{b \in Q}\left|t_{b}\right|$, where $Q$ is a path in $e(\mathcal{E})$ connecting $x_{m}$ and $x_{n}$. Hence, $d_{r}\left(x_{m}, x_{n}\right)<\epsilon$, and $\left\{x_{n}\right\}$ is Cauchy in $\left(\mathcal{N}^{0}, d_{r}\right)$. Moreover, by reversing the roles of $s$ and $r$, we see that $\left\{x_{n}\right\}$ is Cauchy in $\left(\mathcal{N}^{0}, d_{s}\right)$ if it is Cauchy in $\left(\mathcal{N}^{0}, d_{r}\right)$ Consequently, $\left(\widehat{\mathcal{N}^{0}}, d_{r}\right)^{\prime}=\left(\widehat{\mathcal{N}}^{0}, d_{s}\right)^{\prime}$. Similarly, if $r L_{2} s$.

Definition 2.14. In view of Proposition 2.13, we may speak of an $L_{3} s$-terminal. We now give a definition of an $s$-terminal in a component $G$ for a given $s: E(G) \rightsquigarrow$ $[0, \infty]$. For each branch $b$, take $s_{b}^{*}=1$ if $s_{b}=\infty, s_{b}^{*}=s_{b}$ if $s_{b} \in(0, \infty)$, and $s_{b}^{*}>0$ if $s_{b}=0$ with $\sum_{\left\{b: s_{b}=0\right\}} s_{b}^{*}<\infty$. Define an $s$-terminal to be equal to an $s^{*}$-terminal. In particular, this defines a 0 -terminal, and thus ends are simply 0 -terminals by this definition (as well as being $s^{*}$-terminals with $s^{*}>0$ and summable, by Definition 2.1). Also, in Section 4 , we may speak of $R$-terminals since $R_{b}$ may be 0 or $\infty$ for some $b$.

Proposition 2.15. Assume thate is an end in the locally finite graph $\left(\mathcal{B}, \mathcal{N}^{0}\right)$, that $m$ is a positive integer, and that for every finite $\mathcal{E} \subset \mathcal{B}$ there is a finite $\mathcal{F} \subset \mathcal{B}$ with $\mathcal{F} \supset \mathcal{E}$ and with $V e(\mathcal{F}) \cap V\langle\mathcal{F}\rangle$ having no more than m nodes. Given $s: \mathcal{B} \rightsquigarrow(0, \infty)$, there are no more than $m$ s-terminals contained in $e$.

Proof. Suppose, to get a contradiction, that $y_{1}, \ldots, y_{m+1}$ are $s$-terminals with $y_{i} \subset$ $e$ for each $i$. Taking $\epsilon>0$ such that the $B\left(y_{i}, \epsilon\right)$ are pairwise disjoint, we may take $P^{i}=\left\{P_{j}^{i}\right\}_{j=1}^{\infty} \in \mathcal{O}$ with all nodes of $P^{i}$ in $B\left(y_{i}, \epsilon\right)$ and with those nodes converging to $y_{i}$. Take $\mathcal{E}=\left\{P_{1}^{i}: 1 \leq i \leq m+1\right\}$ and $\mathcal{F}$ by hypothesis with $\mathcal{E} \subset \mathcal{F}$ 
and $V e(\mathcal{F}) \cap V\langle\mathcal{F}\rangle$ having no more than $m$ nodes. But, $V e(\mathcal{F}) \cap V\langle\mathcal{F}\rangle$ contains a node of $P^{i}$ for $1 \leq i \leq m+1$ and therefore has no less than $m+1$ nodes, a contradiction.

The proof of Theorem 2.12 showed that each $s$-terminal is a subset of an end when we view these entities as equivalence classes of one-ended paths. We state this as a separate result and extend it.

Proposition 2.16. Let $\left(\mathcal{B}, \mathcal{N}^{0}\right)$ be a connected locally finite graph with $s, r: \mathcal{B} \rightsquigarrow$ $(0, \infty)$ given. If $t+s \geq r \geq 0$ for some summable $t \geq 0$, then the $s$-terminals are subsets of the r-terminals. In particular, they are subsets of the ends.

Proof. We have that $\mathcal{O}_{s} \subset \mathcal{O}_{r}$, for, if $P \in \mathcal{O}_{s}$, then

$$
\sum_{b \in E(P)} r_{b} \leq \sum_{b \in E(P)}\left(s_{b}+t_{b}\right)<\infty .
$$

Also, if $P^{1}$ and $P^{2}$ are in $\mathcal{O}_{s}$ and equivalent (i.e., are in the same $s$-terminal), then, for all $K>0$, there are infinitely many paths $Q$ with $\sum_{b \in E(Q)}\left(s_{b}+t_{b}\right)<K, Q$ connecting nodes in $P^{1}$ and $P^{2}$. Hence, $P^{1}$ and $P^{2}$ are equivalent in $\mathcal{O}_{r}$ (i.e., are in the same $r$-terminal). The last sentence of the theorem follows from Theorem 2.12 and the added assumption that $r$ is summable.

Note. There can be more than one $s$-terminal in an $r$-terminal.

Theorem 2.17. Given a locally finite graph $\left(\mathcal{B}, \mathcal{N}^{0}\right)$, assume that $M$ is an open and closed neighborhood in $\left(\widehat{\mathcal{N}}^{0}, d_{\mathcal{E}}\right)$ of the end $e_{0}$ such that $\left\langle M \cap \mathcal{N}^{0}\right\rangle$ is connected. Then, there exists a finite branch set $\mathcal{F}$ such that

$$
M=V e_{0}(\mathcal{F}) \cup\left\{e \in \partial\left(\mathcal{B}, \mathcal{N}^{0}\right): e(\mathcal{F})=e_{0}(\mathcal{F})\right\}
$$

Proof. We may suppose that $\left(\mathcal{B}, \mathcal{N}^{0}\right)$ is a connected graph $G$. Let

$$
V_{0}=\left\{v \in M \cap \mathcal{N}^{0}: \exists w \in \mathcal{N}^{0} \backslash M \text { with } w \text { adjacent to } v\right\} .
$$

Suppose $V_{0}$ is infinite. Then, there exists a sequence $\left\{v_{n}\right\}$ in $V_{0}$ convergent to some end $e_{1}$. We may take a subsequence $\left\{w_{n_{k}}\right\}$ in $\mathcal{N}^{0} \backslash M$ with $w_{n_{k}}$ adjacent to $v_{n_{k}}$ with $\left\{w_{n_{k}}\right\}$ convergent to an end $e_{2}$. Given $s: \mathcal{B} \rightsquigarrow(0, \infty)$ summable, we have $d_{s}\left(w_{n_{k}}, v_{n_{k}}\right) \rightarrow 0$, and so $e_{1}=e_{2}$. But, $e_{1} \in M$ and $e_{2} \in \widehat{\mathcal{N}^{0}} \backslash M$ since $M$ is open and closed; whence $e_{1} \neq e_{2}$, a contradiction. Thus, $V_{0}$ is finite. Take $\mathcal{F}=\left\langle M \cap \mathcal{N}^{0}, \mathcal{N}^{0} \backslash M\right\rangle$, which is finite since $G$ is locally finite. Note that the graph $\left\langle M \cap \mathcal{N}^{0}\right\rangle$ is infinite and connected and is equal to $e_{0}(\mathcal{F})$. Thus, $M$ is $V e_{0}(\mathcal{F})$ along with all the ends of $e_{0}(\mathcal{F})$.

Remark. It is tempting to use the driving-point resistance (i.e., the input resistance; see, for example, [12]) between pairs of nodes as our metric, at least in the linear case, for it satisfies the triangle inequality as well as the other metric axioms. It has some appealing features; it identifies some $s$-terminals which are forced to have the same potential. But, it does not seem large enough to force easily the continuity of potentials, in contrast to what occurs with $d_{s}(x, y)$. 


\section{Current Flows into $s$-TERMinals}

Given a locally finite digraph $\left(\mathcal{B}, \mathcal{N}^{0}\right)$ and $i: \mathcal{B} \rightsquigarrow \mathbb{R}^{1}$ satisfying Kirchhoff's current law at all nodes $n \in \mathcal{N}^{0}$, we wish to define the flow of $i$ into an $s$-terminal, as a step toward studying Kirchhoff's current law at a 1-node $n^{1}$. The simplest case, as shown by Corollary 3.4, seems to flow into an isolated end. Nonisolated ends and even isolated $s$-terminals all involve limits. We are led to a natural definition for $s$-terminals, which can be applied to existence theorems in Section 4.

Definition 3.1. Let $\left(\mathcal{B}, \mathcal{N}^{0}\right)$ be a locally finite digraph. Let $\mathcal{V}$ and $\mathcal{W}$ be disjoint subsets of $\mathcal{N}^{0}$. Recall that $\langle\mathcal{V}, \mathcal{W}\rangle$ denotes the branches $b$ incident to nodes of both $\mathcal{V}$ and $\mathcal{W}$. Given a real-valued function $i$ defined on a domain that includes $\langle\mathcal{V}, \mathcal{W}\rangle$, define the flow $I(i, \mathcal{V}, \mathcal{W})$ of $i$ from $\mathcal{V}$ into $\mathcal{W}$ to be

$$
\sum_{b \in\langle\mathcal{V}, \mathcal{W}\rangle} i_{b} \epsilon_{b}
$$

if this sum converges absolutely, where $\epsilon_{b}=+1$ if $\partial^{+} b \in \mathcal{V}$ and $\epsilon_{b}=-1$, otherwise. If $\mathcal{V} \cup \mathcal{W}=\mathcal{N}^{0}$, we write $I(i, \mathcal{W})$ for $I\left(i, \mathcal{N}^{0} \backslash \mathcal{W}, \mathcal{W}\right)$

Example 3.2. This example illustrates Definition 3.1. Consider Figure 2. The letters $f, g, \ldots, o$ are names of branches, not the currents in them. The current in, say, branch $x$ is denoted by $i_{x}$. Let $\left(\mathcal{B}, \mathcal{N}^{0}\right)$ be as sketched with ends $a, b$, and $e_{0}$ and with oriented branches $f$ to $o$ as shown. Let $\mathcal{E}_{0}=\{f\}$ and $\mathcal{F}=$ $\{f, g, h, i, j, k, l, m, n, o\}$. We take the ends $e_{0}$ and $b$ to be so defined that $e_{0}\left(\mathcal{E}_{0}\right)$ is the subgraph to the right of $f, e_{0}(\mathcal{F})$ is the subgraph induced by all the nodes above and to the right of the upper dotted curve, and $b(\mathcal{F})$ is the subgraph induced by the nodes below the lower dotted curve. Thus,

$$
\begin{gathered}
I\left(i, V e_{0}\left(\mathcal{E}_{0}\right)\right)=i_{f}, \\
I(i, V b(\mathcal{F}))=i_{g}+i_{k}+i_{m}+i_{n}+i_{o},
\end{gathered}
$$

and

$$
I\left(i, V e_{0}(\mathcal{F})\right)=i_{h}+i_{j}-i_{m}-i_{n}-i_{o}
$$

The next theorem shows how the first current on the left-hand side is the sum of the last two.

Theorem 3.3. Let $e$ be an end in the locally finite digraph $\left(\mathcal{B}, \mathcal{N}^{0}\right)$. Let $\mathcal{E}_{0}$ and $\mathcal{F}$ be finite branch sets with $\mathcal{E}_{0} \subset \mathcal{F}$. Suppose $\left\{B_{1}, \ldots, B_{R}\right\}$ is the set of infinite components of the subgraph $\left(E e\left(\mathcal{E}_{0}\right) \backslash \mathcal{F}, V e\left(\mathcal{E}_{0}\right)\right)$. Then, for $i: \mathcal{B} \rightsquigarrow \mathbb{R}^{1}$ satisfying Kirchhoff's current law at all $n \in \mathcal{N}^{0}$,

$$
\sum_{j=0}^{R} I\left(i, V B_{j}\right)=I\left(i, V e\left(\mathcal{E}_{0}\right)\right) .
$$

Proof. (a) First, suppose that $\mathcal{F} \supset \mathcal{E}_{0} \cup A\left(\mathcal{E}_{0}\right)$. Next, for each branch $b \in \mathcal{F}$ incident to a $u \in V\left(\mathcal{B}_{j}\right)$ and a $v \in V\left(B_{k}\right)$ for $j \neq k$, we introduce an imaginary node $w$ and replace $b$ by two branches $b_{u}$ and $b_{v}$. If $\partial^{+} b=u$, let $\partial^{+} b_{u}=u$ and $\partial^{+} b_{v}=w$, and take the current in each branch to be $i_{b}$. Then, the cuts $\left\langle V B_{i}, \mathcal{N}^{0} \backslash V B_{i}\right\rangle$ and $\left\langle V e\left(\mathcal{E}_{0}\right), \mathcal{N}^{0} \backslash V e\left(\mathcal{E}_{0}\right)\right\rangle$ form mutually disjoint sets. Now, $V e\left(\mathcal{E}_{0}\right) \backslash \bigcup_{j=0}^{R} V B_{j}$ is finite since $\mathcal{F} \backslash \mathcal{E}_{0}$ is finite, and thus there are finitely many finite components of 


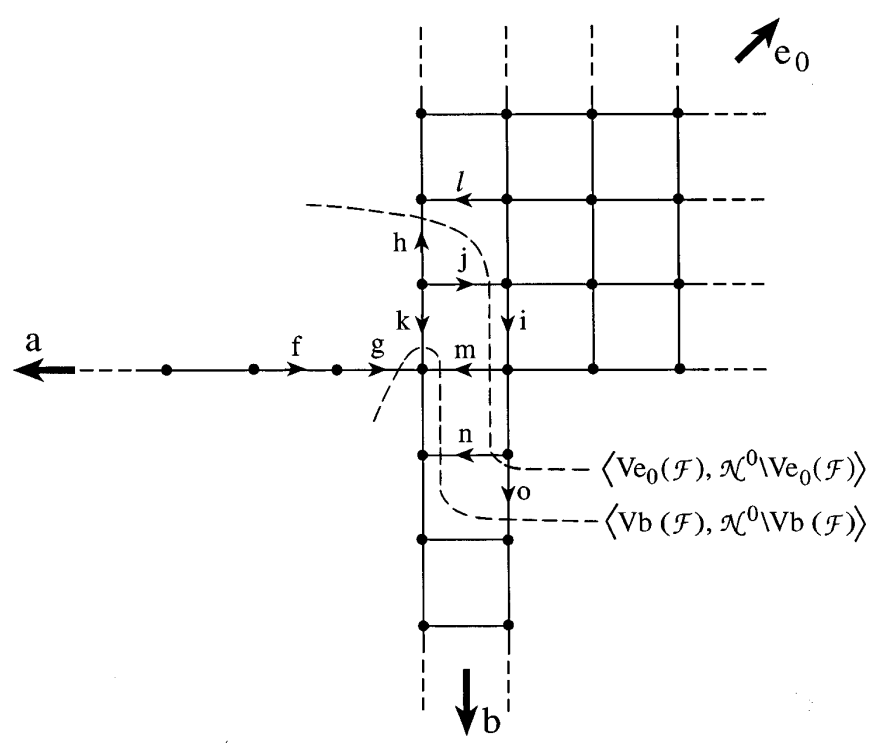

Figure 2. The illustration for Example 3.2. This is a quarterplane grid to which is appended a ladder network extending downward and a one-ended path extending to the left. The upper dotted curve denotes the cut $\left\langle V e_{0}(\mathcal{F}), \mathcal{N}^{0} \backslash V e_{0}(\mathcal{F})\right\rangle$. The lower dotted curve denotes the cut $\left\langle V b(\mathcal{F}), \mathcal{N}^{0} \backslash V b(\mathcal{F})\right\rangle$.

$\left(E e\left(\mathcal{E}_{0}\right) \backslash \mathcal{F}, V e\left(\mathcal{E}_{0}\right)\right)$. Equating the current $I\left(i, V e\left(\mathcal{E}_{0}\right) \backslash \bigcup_{j=0}^{R} V B_{j}\right)$ to zero, we get (3) since we have that

$$
\left\langle V e\left(\mathcal{E}_{0}\right) \backslash \bigcup_{j=0}^{R} V B_{j}, \mathcal{N}^{0} \backslash\left(V e\left(\mathcal{E}_{0}\right) \backslash \bigcup_{j=0}^{R} V B_{j}\right)\right\rangle
$$

is the disjoint union

$$
\left\langle V e\left(\mathcal{E}_{0}\right), \mathcal{N}^{0} \backslash V e\left(\mathcal{E}_{0}\right)\right\rangle \cup\left(\bigcup_{j=0}^{R}\left\langle V B_{j}, \mathcal{N}^{0} \backslash V B_{j}\right\rangle\right) .
$$

(b) Next, assuming that $\mathcal{F} \supset \mathcal{E}_{0}$ but not $\mathcal{F} \supset A\left(\mathcal{E}_{0}\right)$, we take $H$ to be a finite subgraph containing $\mathcal{F} \cup A(\mathcal{F})$, and for each infinite component $B_{j}$ of $e\left(\mathcal{E}_{0}\right) \backslash \mathcal{F}$, we take $\left\{B_{j k}: 0 \leq k \leq R_{j}\right\}$ as the set of infinite components of $B_{j} \backslash H$. By (a), with $B_{j}=e_{j}(\mathcal{F})$ say,

$$
I\left(i, V B_{j}\right)=\sum_{k=0}^{R(j)} I\left(i, V B_{j k}\right),
$$

and so

$$
\sum_{j=0}^{R} I\left(i, V B_{j}\right)=\sum_{j=0}^{R} \sum_{k=0}^{R(j)} I\left(i, V B_{j k}\right) .
$$


But, $\left\{B_{j k}: j=0, \ldots, R ; k=0, \ldots, R(j)\right\}$ is the set of infinite components of $e\left(\mathcal{E}_{0}\right) \backslash H$, and therefore

$$
I\left(i, e\left(\mathcal{E}_{0}\right)\right)=\sum_{j=0}^{R} \sum_{k=0}^{R(j)} I\left(i, B_{j k}\right),
$$

giving (3).

Corollary 3.4. Let $e_{0}$ be an isolated end in the locally finite digraph $\left(\mathcal{B}, \mathcal{N}^{0}\right)$, and suppose that $i: \mathcal{B} \rightsquigarrow \mathbb{R}^{1}$ satisfies Kirchhoff's current law at all $n \in \mathcal{N}^{0}$. Suppose $\mathcal{E}_{0} \subset \mathcal{B}$ is finite and $e \neq e_{0} \Rightarrow e\left(\mathcal{E}_{0}\right) \neq e_{0}\left(\mathcal{E}_{0}\right)$. Then, for $\mathcal{E}$ finite with $\mathcal{E} \supset \mathcal{E}_{0}$, we have $I\left(i, V e_{0}(\mathcal{E})\right)=I\left(i, V e_{0}\left(\mathcal{E}_{0}\right)\right)$.

Definition 3.5. We suppose that $e_{0}$ is an isolated end in the locally finite digraph $\left(\mathcal{B}, \mathcal{N}^{0}\right)$ and that $i: \mathcal{B} \rightsquigarrow \mathbb{R}^{1}$ satisfies Kirchhoff's current law at all $n \in \mathcal{N}^{0}$. The flow $I\left(i, e_{0}\right)$ of $i$ into $e_{0}$ is taken to be $I\left(i, V e_{0}\left(\mathcal{E}_{0}\right)\right)$, where, for all $f \neq e, f\left(\mathcal{E}_{0}\right) \neq e\left(\mathcal{E}_{0}\right)$.

Note. Let $\mathcal{U}\left(e_{0}\right)$ be the set of open neighborhoods of an isolated end $e_{0}$. Then, for $M \in \mathcal{U}\left(e_{0}\right)$ with $M \subset V e_{0}\left(\mathcal{E}_{0}\right) \cup\left\{e_{0}\right\}$, we have $M \cap \mathcal{N}^{0}=V e_{0}(\mathcal{E}) \backslash A$ for some finite $\mathcal{E}$, where $A$ is finite, and therefore $I\left(i, M \cap \mathcal{N}^{0}\right)=I\left(i, V e_{0}(E)\right)$. Now, since $e_{0}$ is isolated, all sufficiently small neighborhoods of $e_{0}$ are both open and closed. Hence, with $\mathcal{U}^{*}\left(e_{0}\right)$ denoting the open and closed neighborhoods $M$ of $e_{0}$ and with $\left\langle M \cap \mathcal{N}^{0}\right\rangle$ connected, we may write

$$
I\left(i, e_{0}\right)=\lim _{M \in \mathcal{U}^{*}\left(e_{0}\right)} I\left(i, M \cap \mathcal{N}^{0}\right)=\lim _{M \in \mathcal{U}\left(e_{0}\right)} I\left(i, M \cap \mathcal{N}^{0}\right),
$$

by Theorem 2.17. This result anticipates Definition 3.10 for the current flow into an $s$-terminal.

Now, we extend this idea to give the flow of $i$ into an end that is a limit point of the set of ends, but an isolated one.

Example 3.6. Let the digraph $G$ be as sketched in Figure 3 with current $i$ satisfying Kirchhoff's current law as shown. For each integer $n$ there is an end $e_{n}$, which is isolated. Then, there are two nonisolated ends $e_{L}$ and $e_{R}$ as indicated. We see that $I\left(i, e_{n}\right)=2^{-n}$ for $n \in \mathbf{N}$ and that $I\left(i, e_{-n}\right)=-2^{-n}$ for $n \in \mathbf{N}, n \neq 0$. It is natural to take $I\left(i, e_{R}\right)=1=\lim _{n \rightarrow \infty} I\left(i, B_{n}\right)$, where $B_{n}$ is the node set of the infinite component on the right obtained by removing the horizontal branch with current $1+2^{-n}$. Similarly, we may set $I\left(i, e_{L}\right)=2$.

Proposition 3.7. Let $e_{0}$ be an end in the locally finite graph $\left(\mathcal{B}, \mathcal{N}^{0}\right)$. Suppose $\mathcal{E}$ and $\mathcal{F}$ are finite branch sets and $E e_{0}(\mathcal{E}) \subset E e_{0}(\mathcal{F})$. Then, $e_{0}\left(\mathcal{F} \cup\left(\mathcal{E} \cap E e_{0}(\mathcal{F})\right)\right)=$ $e_{0}(\mathcal{E})=e_{0}(\mathcal{E} \cup \mathcal{F})$.

Proof. We show that the first equality holds. Write $\mathcal{E}^{*}=\mathcal{F} \cup\left(\mathcal{E} \cap E e_{0}(\mathcal{F})\right)$. Now, $\mathcal{E}^{*} \subset \mathcal{E} \cup \mathcal{F}$, giving $e_{0}(\mathcal{E} \cup \mathcal{F}) \subset e_{0}\left(\mathcal{E}^{*}\right)$, and so $e_{0}\left(\mathcal{E}^{*}\right)$ is the component of $\left(\mathcal{B} \backslash \mathcal{E}^{*}, \mathcal{N}^{0}\right)$ containing $e_{0}(\mathcal{E} \cup \mathcal{F})$. Now, if $b \in E e_{0}(\mathcal{E})$, then $b \in e_{0}(\mathcal{F})$ by hypothesis, implying that $b \notin\left(\mathcal{E} \cap E e_{0}(\mathcal{F})\right) \cup \mathcal{F}=\mathcal{E}^{*}$, and hence $e_{0}(\mathcal{E})$ is contained in some component of $\left(\mathcal{B} \backslash \mathcal{E}^{*}, \mathcal{N}^{0}\right)$. Since $e_{0}(\mathcal{E}) \supset e_{0}(\mathcal{E} \cup \mathcal{F})$, it follows that $e_{0}(\mathcal{E}) \subset e_{0}\left(\mathcal{E}^{*}\right)$.

Similarly, for the reverse inclusion, we want to show that, if $b \in E e_{0}\left(\mathcal{E}^{*}\right)$, then $b \notin \mathcal{E}$. But, such a $b$ is not in $\mathcal{E}^{*}$, hence not in $\mathcal{E} \cap E e_{0}(\mathcal{F})$ by definition of $\mathcal{E}^{*}$. But, $E e_{0}\left(\mathcal{E}^{*}\right) \subset E e_{0}(\mathcal{F})$, so that $e \notin \mathcal{E}$. The second equality is straightforward.

Proposition 3.8. Let $e_{0}$ be an end in the locally finite digraph $\left(\mathcal{B}, \mathcal{N}^{0}\right)$, and let $L \in \mathbb{R}^{1}$. For $i: \mathcal{B} \rightsquigarrow \mathbb{R}^{1}$ the following are equivalent. 


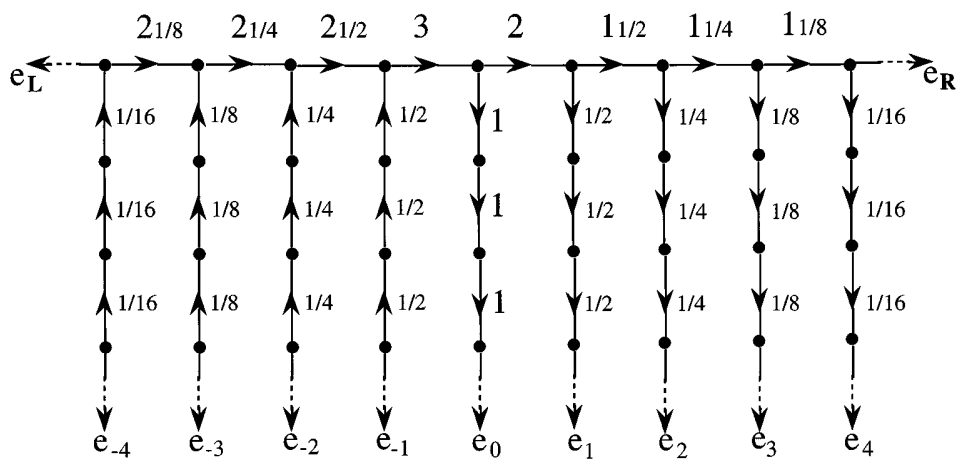

Figure 3. The illustration for Example 3.6. The numbers indicate currents; the letters ends.

(a) The net $\mathcal{E} \mapsto I\left(i, V e_{0}(\mathcal{E})\right)$ converges to $L$, the net being defined on the finite branch sets ordered by inclusion, that is, $\mathcal{E}<\mathcal{F}$ if and only if $\mathcal{E} \subset \mathcal{F}$. In other words, for each $\epsilon>0$, there exists an $\mathcal{E}_{0}$ such that, if $\mathcal{E} \supset \mathcal{E}_{0}$, then $\left|I\left(i, V e_{0}(\mathcal{E})\right)-L\right|<\epsilon$.

(b) The net $e_{0}(\mathcal{E}) \mapsto I\left(i, e_{0}(\mathcal{E})\right)$ converges to $L$ on the components $e_{0}(\mathcal{E})$ with $\mathcal{E}$ finite, where $e_{0}(\mathcal{E})<e_{0}(\mathcal{F})$ if and only if $E e_{0}(\mathcal{E}) \supset E e_{0}(\mathcal{F})$. In other words, for each $\epsilon>0$, there exists an $\mathcal{E}_{0}$ such that, if $E e_{0}(\mathcal{E}) \subset E e_{0}\left(\mathcal{E}_{0}\right)$, then $\left|I\left(i, V e_{0}(\mathcal{E})\right)-L\right|<\epsilon$.

(c) If $\left\{\mathcal{E}_{n}\right\}$ is a sequence of finite branch sets and if the filter base $\left\{V e_{0}\left(\mathcal{E}_{n}\right)\right\}$ converges to $e_{0}$, then $I\left(i, V e_{0}\left(\mathcal{E}_{n}\right)\right) \rightarrow L$.

(d) If $\left\{\mathcal{E}_{n}\right\}$ is a sequence of finite branch sets, if the filter base $\left\{V e_{0}\left(\mathcal{E}_{n}\right)\right\}$ converges to $e_{0}$, and if $\mathcal{E}_{n} \subset \mathcal{E}_{n+1} \subset \mathcal{E}_{n} \cup E e_{0}\left(\mathcal{E}_{n}\right)$ for all $n$, then $I\left(i, V e_{0}\left(\mathcal{E}_{n}\right)\right) \rightarrow L$.

(e) Let $\mathcal{U}^{*}\left(e_{0}\right)$ be the set of open and closed neighborhoods $M$ of $e_{0}$ such that $\left\langle M \cap \mathcal{N}^{0}\right\rangle$ is connected. The net $M \mapsto I\left(i, M \cap \mathcal{N}^{0}\right)$ converges to $L$, the net being defined on $\mathcal{U}^{*}\left(e_{0}\right)$. In other words, for each $\epsilon>0$ there exists an $M_{0} \in \mathcal{U}^{*}\left(e_{0}\right)$ such that, for all $M \subset M_{0}$ with $M \in \mathcal{U}^{*}\left(e_{0}\right)$, we have $\left|I\left(i, M \cap \mathcal{N}^{0}\right)-L\right|<\epsilon$.

Proof. (a) $\Rightarrow$ (b): By Proposition 3.7.

(b) $\Leftrightarrow(\mathrm{e})$ : By Theorem 2.12. (b) $\Rightarrow$ (c): Immediate.

(c) $\Rightarrow$ (d): Nothing to prove.

(d) $\Rightarrow$ (a): If (a) does not hold, there is an $\epsilon_{0}>0$ and an increasing sequence $\left\{\mathcal{E}_{n}\right\}$ with $\bigcup \mathcal{E}_{n}=E G$, where $e_{0}$ is an end in the component $G$ such that $\mid I\left(i, V e_{0}\left(\mathcal{E}_{n}\right)\right)$ $L \mid>\epsilon_{0}$ for all $n$. By Proposition 3.7, replacing each $\mathcal{E}_{n}$ inductively by $\mathcal{E}_{n}^{*}$, where $\mathcal{E}_{1}^{*}=\mathcal{E}_{1}$ and $\mathcal{E}_{n+1}^{*}=\mathcal{E}_{n}^{*} \cup\left(\mathcal{E}_{n+1} \cap E e_{0}\left(\mathcal{E}_{n}^{*}\right)\right)$, we have $\mathcal{E}_{n+1}^{*} \subset \mathcal{E}_{n}^{*} \cup E e_{0}\left(\mathcal{E}_{n}^{*}\right)$ for all $n$. But, $\left|I\left(i, e_{0}\left(\mathcal{E}_{n}^{*}\right)\right)-L\right|>\epsilon_{0}$, contradicting (d).

Definition 3.9. If any and therefore all of (a) to (e) hold and if $i: \mathcal{B} \rightsquigarrow \mathbb{R}^{1}$ satisfies Kirchhoff's current law at every $n \in \mathcal{N}^{0}$, we write $I\left(i, e_{0}\right)=L$ and say that the flow of $i$ into $e_{0}$ is $L$.

We use (e) to extend this definition to the flow of $i$ into an $s$-terminal.

Definition 3.10. Let $\left(\mathcal{B}, \mathcal{N}^{0}\right)$ be a locally finite digraph with $s: \mathcal{B} \rightsquigarrow(0, \infty)$. Let $i: \mathcal{B} \rightsquigarrow \mathbb{R}^{1}$ satisfy Kirchhoff's current law at every $n \in \mathcal{N}^{0}$. For $e$ an $s$-terminal in a component $G$ having countably many $s$-terminals, let $\mathcal{U}^{*}(e)$ denote the set of 
open and closed neighborhoods $M$ of $e$ under $d_{s}$ such that $\left\langle M \cap \mathcal{N}^{0}\right\rangle$ is connected. Assume that $I\left(i, M \cap \mathcal{N}^{0}\right)$ exists for all $M$ in some terminal set of $\mathcal{U}^{*}(e)$, that is, for $\left\{M \in \mathcal{U}^{*}(e): M \subset M^{0}\right\}$, where $M^{0} \in \mathcal{U}^{*}(e)$. We then define the flow $I(i, e)$ of $i$ into $e$ by

$$
I(i, e)=\lim _{M \in \mathcal{U}^{*}(e)} I\left(i, M \cap \mathcal{N}^{0}\right)
$$

if the limit exists.

Note. If $e$ is in the component $G$ and if there are only countably many $s$-terminals in $G$, then $\mathcal{U}^{*}(e)$ is a directed set since the ball $B(e, \epsilon)$ with $\epsilon \neq d(e, f)$ for $f$ an $s$-terminal in $G$ is in $\mathcal{U}^{*}(e)$.

Example 3.11. Let us take $\mathcal{N}^{0}$ to consist of points $p_{m, n}=\left(2^{-m}, 2^{-n}\right)$ for $m, n \in$ $\{0,1,2, \ldots\}=\mathbf{N}$. Take $\mathcal{B}$ to be horizontal and vertical line segments between adjacent nodes $p_{m, n}$ and $p_{m+1, n}$ or between $p_{m, n}$ and $p_{m, n+1}$, as indicated in Figure 4. Take $d=d_{s}$ with $s_{b}$ being the length of $b$. This gives the metric $d$ as the $l_{1}$ distance on $\mathcal{N}^{0}$. We see that

$$
\left(\widehat{\mathcal{N}}^{0}, d\right)^{\prime}=\left\{\left(2^{-k}, 0\right): k \in \mathbf{N}\right\} \cup\left\{\left(0,2^{-k}\right): k \in \mathbf{N}\right\} \cup\{(0,0)\},
$$

and that

$$
\left(\widehat{\mathcal{N}}^{0}, d\right)^{\prime \prime}=\{(0,0)\} .
$$

In Figure 4, we have sketched the ball $B_{1}$ with center at $\left(0,2^{-1}\right)$ and radius about .2 and also the ball $B_{2}$ with center at $(0,0)$ and radius about .26 . This can serve, given current $i$, to illustrate the following.

Proposition 3.12. Suppose that $\left(\mathcal{B}, \mathcal{N}^{0}\right)$ is locally finite and that $d_{s}$ is the metric given by $s: B \rightsquigarrow(0, \infty)$. Suppose $\left(\mathcal{N}^{0}, d_{s}\right)$ is totally bounded. Suppose $i: \mathcal{B} \rightsquigarrow \mathbb{R}^{1}$ satisfies Kirchhoff's current law at every $n \in \mathcal{N}^{0}$.

(a) For $f$ an isolated point of $\widehat{\mathcal{N}}^{\prime}$ and $\mathcal{U}(f)$ the set of neighborhoods of $f$, if there exists $M_{0} \in \mathcal{U}(f)$ such that $\operatorname{cl}\left(M_{0}\right) \cap{\widehat{\mathcal{N}^{0}}}^{\prime}=\{f\}$ and if $I\left(i, M_{0} \cap \mathcal{N}^{0}\right)$ exists, then $I\left(i, M \cap \mathcal{N}^{0}\right)$ exists for all $M \in \mathcal{U}(f)$ with $\operatorname{cl}(M) \cap \widehat{\mathcal{N}}^{\prime}=\{f\}$ and equals $I\left(i, M_{0} \cap \mathcal{N}^{0}\right)$. In particular, $I(i, f)$ exists and equals $I\left(i, M_{0} \cap \mathcal{N}^{0}\right)$.

(b) For $f$, an isolated point of $\widehat{\mathcal{N}}^{\prime \prime}$, if there exists $M_{0} \in \mathcal{U}^{*}(f)$ such that $\operatorname{cl}\left(M_{0}\right) \cap$ $\widehat{\mathcal{N}}^{\prime \prime}=\{f\}$ and if $I\left(i, M_{0} \cap \mathcal{N}^{0}\right)$ exists, then $I\left(i, M \cap \mathcal{N}^{0}\right)$ exists for all $M \in \mathcal{U}^{*}(f)$ such that $M \cap \widehat{\mathcal{N}}^{\prime \prime}=\{f\}$.

(c) For $f$ and $M_{0}$ as in (b), if $\sum I\left(i, e_{j}\right)$ converges absolutely, where the sum is over all isolated $s$-terminals $e_{j}$ in $M_{0}$, then $I(i, f)$ exists, and

$$
I(i, f)=I\left(i, M_{0} \cap \mathcal{N}^{0}\right)-\sum\left\{I(i, e): e \in M_{0} \cap \widehat{\mathcal{N}}^{\prime} \backslash\{f\}\right\} .
$$

Proof. (a) Assume $M \subset M_{0}$. By the compactness of $\widehat{\mathcal{N}}^{0}, \operatorname{cl}\left(M_{0}\right) \backslash M$ is a compact subset of $\mathcal{N}^{0}$, hence finite. Now, let $M_{0} \backslash M=A . M_{0} \cap \mathcal{N}^{0}$ is the disjoint union $A \cup M$. Now, $\left\langle\mathcal{N}^{0} \backslash M_{0}, M_{0} \cap \mathcal{N}^{0}\right\rangle$ is the disjoint union $\left\langle\mathcal{N}^{0} \backslash M_{0}, A\right\rangle \cup\left\langle\mathcal{N}^{0} \backslash M_{0}, M \cap\right.$ $\left.\mathcal{N}^{0}\right\rangle$. Hence,

$$
I\left(i, M_{0} \cap \mathcal{N}^{0}\right)=I\left(i, \mathcal{N}^{0} \backslash M_{0}, A\right)+I\left(i, \mathcal{N}^{0} \backslash M_{0}, M \cap \mathcal{N}^{0}\right) .
$$




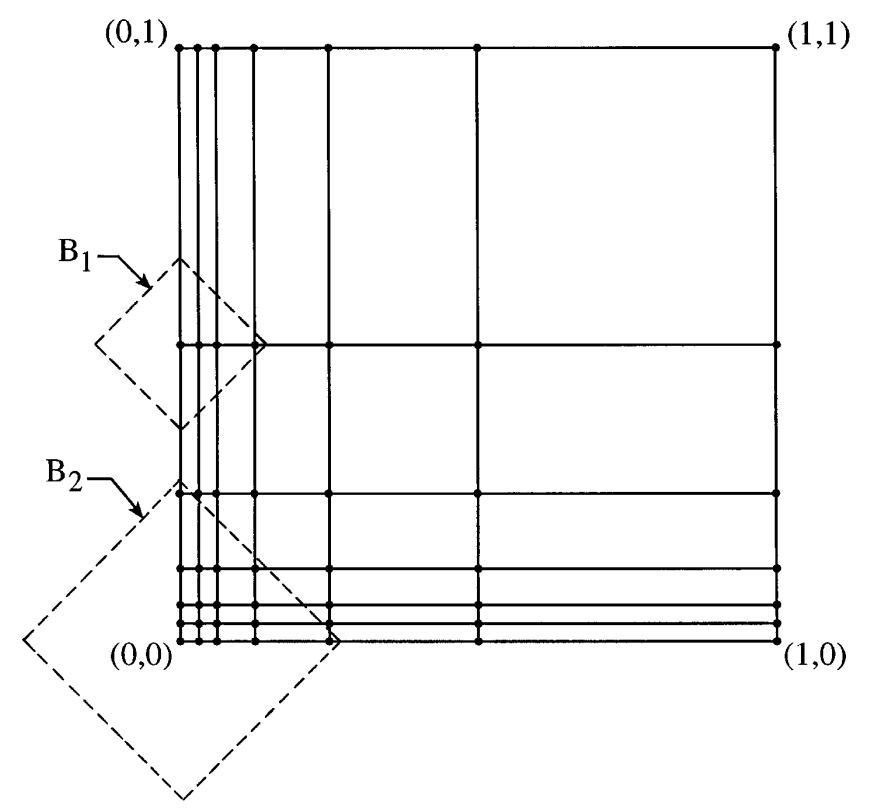

Figure 4. The illustration for Example 3.11. Nodes $p_{m, n}=$ $\left(2^{-m}, 2^{-n}\right)$ are indicated only for $0 \leq m, n \leq 4$. The points $\left(2^{-m}, 0\right)$ and $\left(0,2^{-n}\right)$ are $s$-terminals, and $(0,0)$ is an $s$-terminal that is a limit point of the other $s$-terminals. $B_{1}$ and $B_{2}$ are the balls mentioned in the text.

Note that the left-hand side is equal to $\sum\left\{i_{b} \epsilon_{b}: b \in\left\langle\mathcal{N}^{0} \backslash M_{0}, M_{0} \cap \mathcal{N}^{0}\right\rangle\right\}$, which converges absolutely by Definition 3.1. Hence, the second sum on the right-hand side converges absolutely too, while the first is finite. Also,

$$
\left\langle\mathcal{N}^{0} \backslash M, M \cap \mathcal{N}^{0}\right\rangle=\left\langle\mathcal{N}^{0} \backslash M_{0}, M \cap \mathcal{N}^{0}\right\rangle \cup\left\langle A, M \cap \mathcal{N}^{0}\right\rangle,
$$

which gives

$$
I\left(i, M \cap \mathcal{N}^{0}\right)=I\left(i, \mathcal{N}^{0} \backslash M_{0}, M \cap \mathcal{N}^{0}\right)+I\left(i, A, M \cap \mathcal{N}^{0}\right\rangle .
$$

Now, $I\left(i, \mathcal{N}^{0} \backslash M_{0}, A\right)=I\left(i, A, M \cap \mathcal{N}^{0}\right)$ because $A$ is finite and Kirchhoff's current law holds at all $n \in \mathcal{N}^{0}$. Thus, (4) and (5) give $I\left(i, M \cap \mathcal{N}^{0}\right)=I\left(i, M_{0} \cap \mathcal{N}^{0}\right)$. A rearrangement of this argument shows that, if $I\left(i, M \cap \mathcal{N}^{0}\right)$ exists, then $I\left(i, M_{0} \cap \mathcal{N}^{0}\right)$ exists and has the same value.

Now let us drop the assumption that $M \subset M_{0}$ and merely assume that $M \in \mathcal{U}_{f}$ with $\operatorname{cl}(M) \cap \widehat{\mathcal{N}}^{\prime}=f$, as stated in the hypothesis of (a). Take $M_{1}=M \cap$ $M_{0}$. Then, the above argument shows that $I\left(i, M_{0} \cap \mathcal{N}^{0}\right)=I\left(i, M_{1} \cap \mathcal{N}^{0}\right)$. Also, $I\left(i, M \cap \mathcal{N}^{0}\right)$ exists and equals $I\left(i, M_{1} \cap \mathcal{N}^{0}\right)$. Note, that by taking $M_{0}=B(f, \epsilon)$ with $\operatorname{cl}(B(f, \epsilon)) \cap \widehat{\mathcal{N}}^{\prime}=\{f\}$, ensuring that $M^{*}$ is open and closed and $\left\langle M^{*} \cap\right.$ $\left.\mathcal{N}^{0}\right\rangle$ is connected, we see that $I\left(i, M \cap \mathcal{N}^{0}\right)$ exists for $M \subset M_{0}$ in $\mathcal{U}^{*}(f)$. Thus, $\lim _{M \in \mathcal{U}^{*}(f)} I\left(i, M \cap \mathcal{N}^{0}\right)$ exists. By Definition 3.10, $I(i, f)$ exists. 


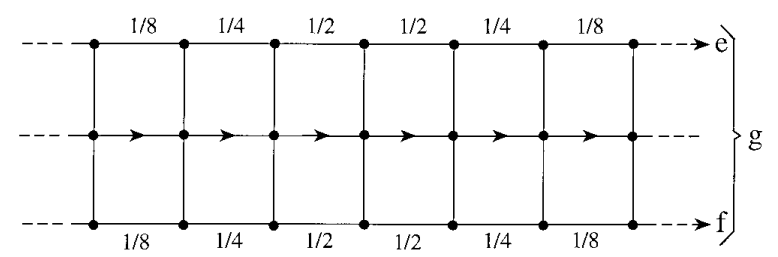

FiguRE 5. The illustration for Remark 3.14. Here, we have a two-way infinite grid. The branch numbers represent resistance values. All unmarked branches have $1 \mathrm{ohm}$ resistances. $e$ and $f$ are $s$-terminals to the right, and $g$ is the end to the right. A current of 1 ampere flows along the middle horizontal path, and current is 0 elsewhere.

(b) Again, suppose $M \subset M_{0}$, as well as $M \in \mathcal{U}^{*}(f)$. Since $\left(\widehat{\mathcal{N}}^{\prime} \cap \operatorname{cl}\left(M_{0}\right)\right) \backslash M$ is compact with no limit points, it is finite, and so $\left(M_{0} \backslash M\right) \cap \widehat{\mathcal{N}}^{\prime}=\left\{e_{1}, \ldots, e_{R}\right\}$, say. Since the $e_{i}$ are in the exterior of $M$, we can take neighborhoods $U_{i}$ of $e_{i}$, which are all disjoint and are subsets of $M_{0} \backslash M$. By compactness, $M_{0}$ is the disjoint union $M \cup\left(\bigcup_{j=1}^{R} U_{j}\right) \cup A$, where $A$ is a finite subset of $\mathcal{N}^{0}$. Thus,

$$
\left\langle\mathcal{N}^{0} \backslash M_{0}, M_{0} \cap \mathcal{N}^{0}\right\rangle=\left\langle\mathcal{N}^{0} \backslash M_{0}, M \cap \mathcal{N}^{0}\right\rangle \cup\left(\bigcup_{j=1}^{R}\left\langle\mathcal{N}^{0} \backslash M_{0}, \mathcal{U}_{j}\right\rangle\right) \cup\left\langle\mathcal{N}^{0} \backslash M_{0}, A\right\rangle .
$$

Arguing as in (a), we obtain

$$
I\left(i, M_{0} \cap \mathcal{N}^{0}\right)=I\left(i, M \cap \mathcal{N}^{0}\right)+\sum_{j=1}^{R} I\left(i, e_{j}\right) .
$$

(c) This follows from (b) and Definition 3.10.

Definition 3.13. In this paper we shall define a 1-node to be a set of question $s$-terminals. This can be construed as a generalized kind of node obtained by electrically shorting together a nonempty set of $s$-terminals. If every $s$-terminal is assigned to a 1-node, that is, if the set of all $s$-terminals is partitioned into 1-nodes, the set of 1 -nodes will be denoted by $\mathcal{N}^{1}$. Furthermore, the triplet $\left(\mathcal{B}, \mathcal{N}^{0}, \mathcal{N}^{1}\right)$ will be called a 1-graph; it will also be called a 1-digraph when all branches have assigned orientations. $\left(\mathcal{B}, \mathcal{N}^{0}, \mathcal{N}^{1}\right)$ will be called locally finite when all nodes in $\mathcal{N}^{0}$ have finite degrees and when all 1-nodes are finite sets.

Remark 3.14. In Example 2.2 there are two $s$-terminals $e$ and $f$ in the single end; call that end $g$. Given $i$ satisfying Kirchhoff's current law at all nodes $n \in \mathcal{N}^{0}$, we have

$$
I(i, e)+I(i, f)=I(i, g) .
$$

The question arises: Given a connected digraph $\left(\mathcal{B}, \mathcal{N}^{0}\right)$ with $s: \mathcal{B} \rightsquigarrow(0, \infty)$ and just two $s$-terminals $e$ and $f$ in the end $g$, does (6) hold? Consider the counterexample illustrated in Figure 5. Let $s_{b}=1$ for all branches except for those in the top and bottom horizontal paths, wherein $s_{b}$ has the values indicated. Take $i_{b}$ to 
be 1 for every branch $b$ in the central horizontal path; all other branch currents are 0 . Then, $I(i, e)=I(i, f)=0$, but $I(i, g)=1$

Proposition 3.15. Suppose $\left(\mathcal{B}, \mathcal{N}^{0}\right)$ is a digraph, $s: \mathcal{B} \rightsquigarrow(0, \infty)$ is given, $g$ is an isolated end, and there are just $K$ s-terminals $e_{1}, \ldots, e_{K}$ in $g$. Suppose for $\epsilon>0$ there is a finite branch set $\mathcal{E} \subset \mathcal{B}$ such that, for all $x \in \operatorname{Vg}(\mathcal{E}), d_{s}\left(x, e_{k}\right)<\epsilon$ for some $k \in\{1, \ldots, K\}$. Suppose $i: \mathcal{B} \rightsquigarrow \mathbb{R}$ satisfies Kirchhoff's current law at all $x \in \mathcal{N}^{0}$, and $I\left(i, e_{k}\right)$ exists for $k=1, \ldots, K$. Then, $I(i, g)=\sum_{k=1}^{K} I\left(i, e_{k}\right)$.

Proof. Take a finite branch set $\mathcal{F}$ such that $g$ is the only end in $g(\mathcal{F})$. Take $\epsilon>0$ such that, for $1 \leq k \leq K, \mathcal{N}^{0} \cap B\left(e_{k}, \epsilon\right)$ are disjoint subsets of $V g(\mathcal{F})$. Take $\mathcal{E} \subset$ $E g(\mathcal{F}), \mathcal{E}$ finite, such that $V g(\mathcal{E}) \subset \bigcup_{k=1}^{K} B\left(e_{k}, \epsilon\right)$. Since there is only one end in $g(\mathcal{F}), g(\mathcal{E})$ is the only infinite component of $(E g(\mathcal{F}) \backslash \mathcal{E}, V g(\mathcal{F}))$, and there are only finitely many nodes of $g(\mathcal{F})$ that are not of $g(\mathcal{E})$. Thus, $A \equiv V g(\mathcal{F}) \backslash \bigcup_{k=1}^{K} B\left(e_{k}, \epsilon\right)$ is finite. Since $I(i, A)=0$ and $V g(\mathcal{F})$ is the disjoint union of $A$ and the $B\left(e_{k}, \epsilon\right)$, we have $I(i, V g(\mathcal{F}))=\sum_{k=1}^{K} I\left(i, V B\left(e_{k}, \epsilon\right) \cap \mathcal{N}^{0}\right)$ because these exist according to Proposition 3.12(a). That is, $I(i, g)=\sum I\left(i, e_{k}\right)$.

Definition 3.16. Let $\left(\mathcal{B}, \mathcal{N}^{0}, \mathcal{N}^{1}\right)$ be a locally finite digraph, let $n^{1} \in \mathcal{N}^{1}$, and let $i: \mathcal{B} \rightsquigarrow \mathbb{R}^{1}$ satisfy Kirchhoff's current law at every $n \in \mathcal{N}^{0}$. We shall say that $i$ satisfies Kirchhoff's current law at $n^{1}$ if $I(i, e)$ exists for each $e \in n^{1}$ and if $\sum_{e \in n^{1}} I(i, e)=0$.

We should point out that our present 1-nodes are specialized cases of the 1-nodes defined in [18] and [20]. According to that earlier definition, a 1-node $n^{1}$ is any set of equivalence classes of one-ended paths along with no more than one node of $\mathcal{N}^{0}$, called the embraced 0 -node of $n^{1}$; also, two one-ended paths are considered equivalent if they differ on no more than finitely many branches. Thus, $n^{1}$ as defined earlier can be chosen quite arbitrarily by choosing (finitely or infinitely many) equivalence classes of one-ended paths. On the other hand, in this paper an $s$-terminal is a certain set of one-ended paths as indicated in Corollary 2.4, and therefore our present 1-nodes are more restricted. In particular, the $s$-terminals are determined by the graph and the chosen function $s$. It is only the graph, the function $s$, and the assignment of the $s$-terminals to the 1-nodes that we can choose arbitrarily.

Moreover, if $n^{1} \in \mathcal{N}^{1}$ and $n^{0} \in \mathcal{N}^{0}$, in this paper we have not allowed $n^{0} \in n^{1}$. In [18], [19], and [20], this was allowed, and $n^{0}$ was called an embraced 0-node. Nonetheless, we can now append (no more than) one $n^{0} \in \mathcal{N}^{0}$ as an embraced 0node of any $n^{1}=\left\{e_{1}, \ldots, e_{R}\right\} \in \mathcal{N}^{1}$ through the artifice of appending a one-ended path $P$ of shorts (i.e., branches not in $\mathcal{B}$ ) connecting $n^{0}$ and $f$, where $f$ is the limit of the nodes of $P$. Each short has a current-voltage characteristic that coincides with the $i$-axis $(v=0)$. Then, the $s$-terminal $f$ given by $P$ can be added as another member of $n^{1}$. In this case, Kirchhoff's current law is taken to be

$$
\sum_{j=1}^{R} I\left(i, e_{j}\right)+I(i, f)=0,
$$

where $P$ is in the $s$-terminal $f$. 


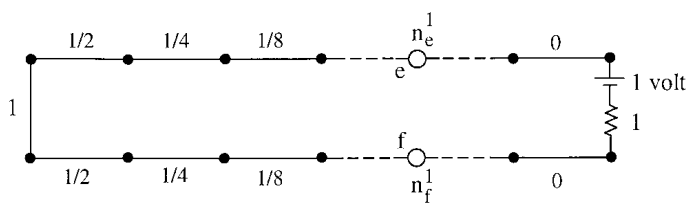

Figure 6(a). An illustration for Example 4.1(a). This is an endless path with two ends $e$ and $f$, to which is connected a 1-volt source through a series resistance of $1 \mathrm{ohm}$. That source branch is connected to $e$ and $f$ through two one-ended paths of shorts - the artifice mentioned at the end of Section 3. $n_{e}^{1}$ and $n_{f}^{1}$ are 1-nodes consisting of the ends as shown. The other branch numbers are branch resistances.

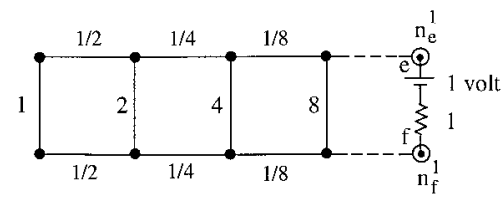

Figure 6(b). An illustration for Example 4.1(b). Here, the endless path of Figure 6(a) is converted into a ladder by appending vertical resistances of $2^{k}$ ohms $(k=0,1,2, \ldots)$. Now, the two ends $e$ and $f$ of Figure 6(a) become $R$-terminals, where $R$ is determined by the resistances.

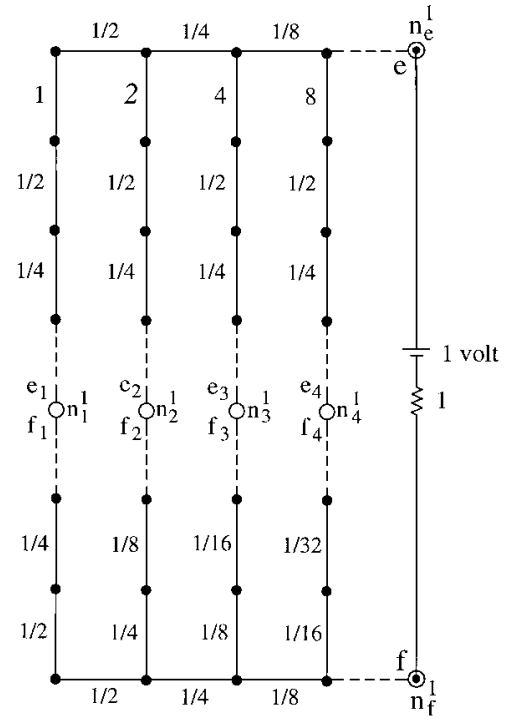

Figure 6(c). An illustration for Example 4.1(c). Here, each vertical branch of Figure 6(b) is augmented by inserting two one-ended paths in series with that branch, the paths being shorted together at their ends through a 1-node. Again the branch numbers denote branch resistances, except for the 1 volt source. 


\section{EXISTENCE OF AN OPERATING POINT}

In this section we establish the existence of an operating point through Theorems $4.4,4.5$, and 4.8; these are modifications of each other. We will start with a monotone network $\mathbf{M}$ on the digraph $\left(\mathcal{B}, \mathcal{N}^{0}\right)$, and shortly we will review the concepts we shall use. Then, we form the $R$-terminals, where $R$ represents for each branch $b$ the Lipschitz constant $R_{b}$ on a certain interval of the branch's $(i, v)$ characteristic curve $M_{b}$. Next, from the $R$-terminals we form 1-nodes as in Section $3 . \mathcal{N}^{1}$ will denote the set of 1-nodes. Our definition of a solution will impose Kirchhoff's current law at all nodes and 1-nodes, as well as Kirchhoff's voltage law through a potential function whose continuity will extend that law from $\mathcal{N}^{0}$ onto $\mathcal{N}^{0} \cup \mathcal{N}^{1}$.

We have the conflicting desires of giving simple results or giving general ones. Let us present three examples to motivate and illustrate our results in a very simple way.

Example 4.1 (a). Refer to Figure 6(a). The component on the left, (i.e., the endless path to the left of the small circles representing the 1-nodes $n_{e}^{1}$ and $n_{f}^{1}$ ) has linear resistances as shown and two ends $e$ and $f$ contained in $n_{e}^{1}$ and $n_{f}^{1}$. The component to the right of the small circles contains a source branch consisting of a voltage source of 1 volt in series with a $1 \mathrm{ohm}$ resistor; that branch, by the artifice described at the end of the last section, is connected to $n_{e}^{1}$ and $n_{f}^{1}$ through two one-ended paths of shorts. Theorem 4.4 gives the existence of a solution here.

Example 4.1 (b). Now, consider Figure 6(b). The component on the left is a ladder with resistances as shown. Note that the horizontal resistances are summable and that there are two $R$-terminals $e$ and $f$. Theorem 4.5 now gives the existence of a solution since the vertical conductances are summable. In this figure we have chosen to show the component on the right as a branch with its extremities (i.e., its elementary tips [20, page 9]) shorted to $e$ and $f$, as in the diagrams of [18] and $[20]$.

Example 4.1 (c). In Figure 6(c), the component on the left of $n_{e}^{1}$ and $n_{f}^{1}$ has resistances as shown. Note that the top vertical conductances are summable. Here, the $R$-terminals $e_{k}$ and $f_{k}$ are shorted to give the 1 -node $n_{k}^{1}$ for each $\mathrm{k}$. Note that $f_{n} \rightarrow f$ as $n \rightarrow \infty ; f$ is not isolated. A solution exists according to Theorem 4.8.

Let us mention that, although we will consider only monotone networks in this paper, our results can be extended to nonmonotone networks through the results of $[2]$.

We now give some definitions concerning a nonlinear monotone network, which are a little simpler than those given by Calvert in [1] and by Minty in [13] because we are aiming at simplicity. For example, we rule out a pure voltage or current source.

We say that a monotone network $\mathbf{M}$ on a digraph $\left(\mathcal{B}, \mathcal{N}^{0}\right)$ is a function that assigns to every $b \in \mathcal{B}$ a maximal monotone function $M_{b}: \mathbb{R} \rightsquigarrow 2^{\mathbb{R}}$, or equivalently a subset $M_{b}$ of $\mathbb{R}^{2}$. We recall that a function $f: \mathbb{R} \rightsquigarrow 2^{\mathbb{R}}$ is monotone when

$$
\left(f(x)^{*}-f(y)^{*}\right)(x-y) \geq 0
$$

where $f(x)^{*}$ is a number in $f(x)$ and $f(y)^{*}$ is a number in $f(y)$. We may write $(x, y) \in f$ to mean $y \in f(x)$. We define $f^{-1}: \mathbb{R} \rightsquigarrow 2^{\mathbb{R}}$ by the statement: For $(x, y) \in \mathbb{R}^{2},(y, x) \in f^{-1}$ if and only if $(x, y) \in f$. We say that $f: \mathbb{R} \rightsquigarrow 2^{\mathbb{R}}$ is 
maximal monotone if the two conditions $\left(x_{1}, y_{1}\right) \in \mathbb{R}^{2}$ and $\left(y-y_{1}\right)\left(x-x_{1}\right) \geq 0$ for all $(x, y) \in f$ imply that $\left(x_{1}, y_{1}\right) \in f$.

We will say that $\mathbf{M}$ satisfies $(A)$ to mean that the following three conditions hold.

(1) For all $b \in \mathcal{B}$, there is at least one $y$ with $(0, y) \in M_{b}$ (i.e., $0 \in \operatorname{Domain}\left(M_{b}\right)$ ), in which case we set

$$
\delta_{b}^{\prime \prime}\left(0,0, M_{b}\right)=\min \left\{|y|:(0, y) \in M_{b}\right\} .
$$

(2) For all $b \in \mathcal{B}$, there is at least one $x$ with $(x, 0) \in M_{b}$ (i.e., $0 \in \operatorname{Range}\left(M_{b}\right)$ ), in which case we set

$$
\delta_{b}^{\prime}\left(0,0, M_{b}\right)=\min \left\{|x|:(x, 0) \in M_{b}\right\} .
$$

$$
\mathbb{I} \equiv \sum_{b \in \mathcal{B}} \delta_{b}^{\prime}\left(0,0, M_{b}\right)<\infty
$$

and

$$
\mathbb{V} \equiv \sum_{b \in \mathcal{B}} \delta_{b}^{\prime \prime}\left(0,0, M_{b}\right)<\infty .
$$

We now state the form of Minty's theorem that we shall use.

Minty's Theorem [13]. Let $\mathbf{M}$ be a monotone network on the finite digraph $\left(\mathcal{B}, \mathcal{N}^{0}\right)$. Suppose that, for all $b \in \mathcal{B}, 0 \in \operatorname{Domain}\left(M_{b}\right)$ and $0 \in \operatorname{Range}\left(M_{b}\right)$. With $\mathbb{I}$ and $\mathbb{V}$ given by $(7)$ and $(8)$, there exists a $\left(i_{b}, v_{b}\right) \in M_{b}$ for each $b \in M_{b}$ with $i$ satisfying Kirchhoff's current law, $v$ satisfying Kirchhoff's voltage law, and with $\left|i_{b}\right| \leq \mathbb{I}$ and $\left|v_{b}\right| \leq \mathbb{V}$ for all $b \in \mathcal{B}$.

That $R_{b}$ and $G_{b}$ are given by $(B)$ is understood to mean that the following two conditions hold.

(i) $R_{b}$ is the Lipschitz constant of $M_{b}$ on $[-\mathbb{I}, \mathbb{I}]$ if $M_{b}$ is a Lipschitz continuous function; otherwise, $R_{b}=\infty$.

(ii) $G_{b}$ is the Lipschitz constant of $M_{b}^{-1}$ on $[-\mathbb{V}, \mathbb{V}]$ if $M_{b}^{-1}$ is a Lipschitz continuous function; otherwise, $G_{b}=\infty$.

Definition 4.2. Let $\mathbf{M}$ be a monotone network on the locally finite digraph $\left(\mathcal{B}, \mathcal{N}^{0}\right)$, and let $\mathcal{N}^{1}$ be a partition of the set of $s$-terminals, where $s: \mathcal{B} \rightsquigarrow[0, \infty]$ is given. We call each element of $\mathcal{N}^{1}$ a 1-node.

We will refer to triple $\left(\mathcal{B}, \mathcal{N}^{0}, \mathcal{N}^{1}\right)$ as an $s$-terminal generated 1-digraph or simply as a terminal generated 1-digraph when $s$ is understood. When every member of every 1-node is an end, we say that $\left(\mathcal{B}, \mathcal{N}^{0}, \mathcal{N}^{1}\right)$ is end generated. As was stated in Section 3, we call $\left(\mathcal{B}, \mathcal{N}^{0}, \mathcal{N}^{1}\right)$ locally finite when every node in $\mathcal{N}^{0}$ has finite degree and every 1 -node is a finite set of terminals.

Note. We will take $s$ to be 0 in Theorem 4.4 and $s=R$ in Theorems 4.5 and 4.8.

Definition 4.3. Let $\mathbf{M}$ be a monotone network on the locally finite digraph $\left(\mathcal{B}, \mathcal{N}^{0}\right)$. Let $s: \mathcal{B} \rightsquigarrow[0, \infty]$ be given. Let $\left(\mathcal{B}, \mathcal{N}^{0}, \mathcal{N}^{1}\right)$ be $s$-terminal generated and locally finite. A solution of $\mathbf{M}$ on $\left(\mathcal{B}, \mathcal{N}^{0}, \mathcal{N}^{1}\right)$ consists of an ordered pair $(i, v)$, where $i: \mathcal{B} \rightsquigarrow \mathbb{R}$ satisfies Kirchhoff's current law at all $n \in \mathcal{N}^{0} \cup \mathcal{N}^{1}$, and there is a continuous $p: \mathcal{N}^{0} \cup \mathcal{N}^{1} \rightsquigarrow \mathbb{R}$ with $p$ restricted to $\mathcal{N}^{0}$ being a potential for $v$ such that, for all $b \in \mathcal{B},\left(i_{b}, v_{b}\right) \in M_{b}$. 


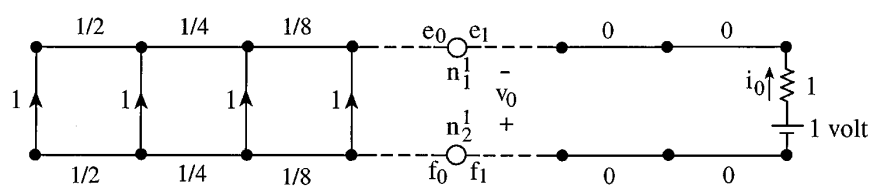

FiguRe 7. A ladder network connected through two 1-nodes $n_{1}^{1}$ and $n_{2}^{1}$ to an endless path, all of whose branches are shorts except for one source branch. All branch numbers other than the 1-volt source represent resistances. $e_{0}, e_{1}, f_{0}$, and $f_{1}$ denote $r$-terminals. Also, $n_{1}^{1}=\left\{e_{0}, e_{1}\right\}$ and $n_{2}^{1}=\left\{f_{0}, f_{1}\right\}$. The arrows denote branch orientations.

Theorem 4.4. Let $\mathbf{M}$ be a monotone network satisfying $(A)$ on the locally finite digraph $\left(\mathcal{B}, \mathcal{N}^{0}\right)$. Let $\left(\mathcal{B}, \mathcal{N}^{0}, \mathcal{N}^{1}\right)$ be end generated and locally finite. Suppose all ends are isolated and, for each end $e$ and $\epsilon>0$, there is a finite branch set $\mathcal{E}$ such that, for $n \in V e(\mathcal{E})$, there is a one-ended path $P$ connecting $n$ and $e$ in $e(\mathcal{E})$ with $\sum_{b \in P} R_{b}<\epsilon$, where $R_{b}$ is given by $(B)$. Then, there exists a solution $(i, v)$ of $\mathbf{M}$ with $\left|i_{b}\right| \leq \mathbb{I}$ and $\left|v_{b}\right| \leq \mathbb{V}$ for every branch $b$.

Note. Making the set of ends finite will slightly simplify the result. Furthermore, if $R$ were summable, every $R$-terminal would be an end, and our condition on $P$ would be automatically satisfied; this would be another simplification. We omit the proof of Theorem 4.4 and Theorem 4.5 because they are just modifications of the proof of Theorem 4.8.

We continue to aim for simplicity, and therefore in Theorem 4.5 we give an existence result in which $\left(\mathcal{B}, \mathcal{N}^{0}, \mathcal{N}^{1}\right)$ is $R$-terminal generated and locally finite and $\mathcal{N}^{1}$ is finite, i.e., there are only a finite number of $R$-terminals. $\emptyset$.

In a later result, Theorem 4.8, we allow elements of $\widehat{\mathcal{N}}^{\prime \prime}$, but require that $\widehat{\mathcal{N}}^{\prime \prime \prime}=$

Theorem 4.5. Let $\mathbf{M}$ be a monotone network on the locally finite digraph $\left(\mathcal{B}, \mathcal{N}^{0}\right)$, satisfying $(A)$. Let $R_{b}$ and $G_{b}$ be given by $(B)$. Let $s_{b}=R_{b}^{*}$ as in Definition 2.14.

(i) Suppose each component $G$ of $\left(\mathcal{B}, \mathcal{N}^{0}\right)$ has $\left(V(G), d_{s}\right)$ totally bounded.

(ii) Assume there are only finitely many R-terminals. Let $\mathcal{N}^{1}$ be a partition of the set of those terminals.

(iii) Suppose that, for each $R$-terminal $f$, there is an $\epsilon>0$ such that $\epsilon<d\left(f, \widehat{\mathcal{N}}^{0} \backslash\{f\}\right)$ and a cofinite subset $C(f)$ of $\left\langle\mathcal{N}^{0} \backslash B(f, \epsilon), B(f, \epsilon) \cap \mathcal{N}^{0}\right\rangle$ such that $\sum_{b \in C(f)} G_{b}<$ $\infty$.

Then, there is a solution $(i, v)$ of $\mathbf{M}$ with $\left|i_{b}\right| \leq \mathbb{I}$ and $\left|v_{b}\right| \leq \mathbb{V}$ for every branch $b$.

Proposition 4.6. There exist monotone networks satisfying $(A)$ and also (i) and (ii), but not (iii), of Theorem 4.5, where $R_{b}$ and $G_{b}$ are given by $(B)$, for which a solution does not exist.

Proof. Consider the linear network sketched in Figure 7. Let $i_{0}$ be the indicated current in the source branch and thereby in the shorts as well. Then $v_{0}=i_{0}-1$ is the indicated voltage drop from $n_{2}^{1}$ to $n_{1}^{1}$. There are just two possibilities we have to consider.

First, assume that $v_{0}=0$. Then, if Kirchhoff's laws are to be satisfied within the ladder, all the branch currents and branch voltages therein must be zero because 
there are no sources within the ladder. Now, choose $0<\epsilon<1$. Then, $B\left(f_{0}, \epsilon\right)$ consists only of $f_{0}$ and nodes along the lower horizontal line of the ladder, and $I\left(i, f_{0}\right)=0$. On the other hand, $B\left(f_{1}, \epsilon\right)$ consists of $f_{1}$ and all the nodes along the lower one-ended path of shorts, and $I\left(i, f_{1}\right)=i_{0}=1$. Thus, Kirchhoff's current law is violated at $n_{2}^{1}$.

Secondly, assume that $v_{0} \neq 0$. Then, if the potential function is to be continuous at $n_{1}^{1}$ and $n_{2}^{1}$, the current flow out of $B\left(f_{0}, \epsilon\right)$ with $\epsilon<1$ again (that is, the sum of currents in the branches of $\left\langle\mathcal{N}_{L}^{0} \backslash B\left(f_{0}, \epsilon\right), B\left(f_{0}, \epsilon\right) \cap \mathcal{N}_{L}^{0}\right\rangle$, where $\mathcal{N}_{L}^{0}$ is the set of all ladder nodes) is infinite. So again, Kirchhoff's current law is violated at $n_{2}^{1}$.

Altogether, no solution for this network exists.

Remark. We do not have uniqueness under the setup of Theorem 4.4. For example, let $\left(\mathcal{B}, \mathcal{N}^{0}, \mathcal{N}^{1}\right)$ be as sketched in Figure 8 ; it is a series connection of infinitely but countably many disjoint endless paths $P_{k}$ connecting the 1-nodes $n_{k}^{1}$ and $n_{k+1}^{1}$. Suppose the sum of the resistances in all the branches is finite. Then, any current (the same in all branches) gives a solution. Thus, any such solution is not unique. However, uniqueness will hold at least under the next hypothesis.

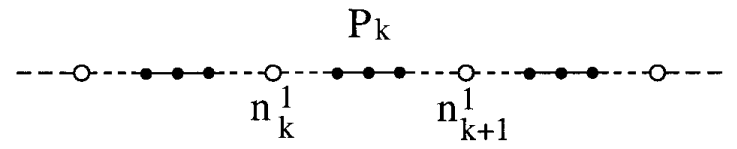

Figure 8. An illustration for the Remark before Theorem 4.7.

This is an endless path of series-connected endless paths.

Theorem 4.7. Under the conditions of Theorem 4.5, suppose that, for all $b, M_{b}$ has no vertical or horizontal segments (i.e., if $\left(x, x^{*}\right) \in M_{b},\left(y, y^{*}\right) \in M_{b}$, and $\left(x^{*}-y^{*}\right)(x-y)=0$, then $x=y$ and $\left.x^{*}=y^{*}\right)$. Then, there is only one solution.

Proof. Suppose $(i, v)$ and $(i+\Delta i, v+\Delta v)$ are solutions. Orient every branch so that $\Delta v \geq 0$. By the strict monotonicity of every $M_{b}$, either $\Delta v_{b}>0$ and $\Delta i_{b}>0$ or $\Delta v_{b}=0$ and $\Delta i_{b}=0$. Also, $\Delta i$ satisfies Kirchhoff's current law at every member of $\mathcal{N}^{0} \cup \mathcal{N}^{1}$. Moreover, there will be a potential function $\Delta p$ that generates $\Delta v$.

Suppose there is a branch $a$ with $\Delta v_{a}>0$ and $\Delta i_{a}>0$. Let $f_{1}, \ldots, f_{n}$ be the finitely many terminals of the network. We can choose neighborhoods $U\left(f_{k}\right)$ around each $f_{k}$ that are so small that they are pairwise disjoint and do not contain the nodes of $a$. Choose the real number $\lambda$ such that $\lambda \in\left(\Delta p\left(\partial^{+} a\right), \Delta p\left(\partial^{-} a\right)\right)$ and $\lambda \neq \Delta p\left(f_{k}\right)$ for all terminals $f_{k}$. This can be done because there are only finitely many terminals. Let $\mathcal{M}=\left\{x \in \mathcal{N}^{0} \cup \mathcal{N}^{1}: \Delta p(x)<\lambda\right\}$. The flow $I$ of $\Delta i$ into $\mathcal{M} \cap \mathcal{N}^{0}$ from $\mathcal{N}^{0} \backslash\left(\mathcal{M} \cap \mathcal{N}^{0}\right)$ is defined according to Definition 3.10 because $\mathcal{M}$ is the union of finitely many disjoint neighborhoods $U\left(f_{k}\right)$ and a finite set of nodes. By Kirchhoff's current law, $I=0$. On the other hand, if branch $b$ is incident to a node $n_{1}$ in $\mathcal{M}$ and a node $n_{2}$ in $\mathcal{N}^{0} \backslash \mathcal{M}$, then $i_{b}>0$ because $\Delta p\left(n_{1}\right)<\lambda \leq \Delta p\left(n_{2}\right)$. Consequently, $I \geq i_{a}>0$. We have a contradiction.

Theorem 4.8. Let $\mathbf{M}$ be a monotone network on the locally finite digraph $\left(\mathcal{B}, \mathcal{N}^{0}\right)$ satisfying $(A)$. Let $R_{b}$ and $G_{b}$ be given by $(B)$. Suppose $\left(\mathcal{B}, \mathcal{N}^{0}, \mathcal{N}^{1}\right)$ is $R$-terminal generated (i.e., $s=R^{*}$; see Definition 2.14) and is locally finite.

(i) Suppose each component of $\left(\mathcal{N}^{0}, d_{s}\right)$ is totally bounded. 
(ii) Suppose $\widehat{\mathcal{N}}^{\text {'II }}$ is empty. Suppose also that there does not exist a sequence $\left\{f_{n}\right\}_{n=1}^{\infty}$ of isolated terminals converging to a terminal $e_{0} \in \widehat{\mathcal{N}}^{\prime \prime}$ with another sequence $\left\{e_{n}\right\}_{n=1}^{\infty} \subset \widehat{\mathcal{N}}^{\prime \prime}$ such that, for every $n, e_{n}$ and $f_{n}$ are in the same 1-node (i.e., are shorted together).

(iii) Suppose that, for each isolated terminal $e$, there is an $\epsilon=\epsilon(e)>0$ with $\epsilon(e)<d_{s}\left(e, \widehat{\mathcal{N}}^{\prime} \backslash\{e\}\right)$ such that

$$
G_{e, \epsilon}=\sum\left\{G_{b}: b \in\left\langle\mathcal{N}^{0} \backslash B(e, \epsilon), B(e, \epsilon) \cap \mathcal{N}^{0}\right\rangle\right\}<\infty
$$

(iv) For each terminal $e \in \widehat{\mathcal{N}}^{\prime \prime}$ there is an $\epsilon=\epsilon(e) \in\left(0, d_{s}\left(e, \widehat{\mathcal{N}}^{\prime \prime} \backslash\{e\}\right)\right.$ ) and a cofinite subset $D$ of $\left\langle\mathcal{N}^{0} \backslash B(e, \epsilon), B(e, \epsilon) \cap \mathcal{N}^{0}\right\rangle$ such that $\sum_{b \in D} G_{b}<\infty$.

(v) For $e_{0} \in \widehat{\mathcal{N}}^{\prime \prime}$ and $\left\{e_{n}\right\}_{n=1}^{\infty}$ a sequence of isolated terminals converging to $e_{0}$ with $e_{n}$ in the 1-node $x_{n}$ for each $n$, we have

$$
\sum_{n=1}^{\infty} \sum_{e \in x_{n} \backslash\left\{e_{n}\right\}} G_{e, \epsilon(e)}<\infty
$$

where $G_{e, \epsilon(e)}$ is defined in (iii).

Then, there exists a solution $(i, v)$ of $\mathbf{M}$ on $\left(\mathcal{B}, \mathcal{N}^{0}, \mathcal{N}^{1}\right)$ with $\left|i_{b}\right| \leq \mathbb{I}$ and $\left|v_{b}\right| \leq \mathbb{V}$ for all branches $b$.

Note. To sum the currents into the $e_{n}$ in (v), we assumed (iii), and so this is not quite a generalization of Theorem 4.5 since we can't just sum over a cofinite set in (iii). Nonetheless, as was noted above, Theorems 4.4 and 4.5 require only small modifications of the proof of Theorem 4.8.

Proof. We may assume that $\left(\mathcal{B}, \mathcal{N}^{0}, \mathcal{N}^{1}\right)$ is what we call 1 -connected, that is, for any terminals $e$ and $f$ there is a finite sequence $\left\{G_{1}, \ldots, G_{K}\right\}$ of distinct components of $\left(\mathcal{B}, \mathcal{N}^{0}\right)$ with $e$ a terminal in $G_{1}, f$ a terminal in $G_{K}$, and, if $K>1$, there is a terminal $r_{j}$ in $G_{j}$ and a terminal $l_{j}$ in $G_{j+1}$ in the same 1-node for $j=1$ to $K-1$. (Corollary 2.6 is the motivation for this.) We will say, equivalently, that $e$ and $f$ are 1 -connected by the sequence $\left\{r_{1}, l_{1}, r_{2}, \ldots, l_{K}\right\}$. Now, a solution in each maximally 1-connected subgraph of $\left(\mathcal{B}, \mathcal{N}^{0}, \mathcal{N}^{1}\right)$ gives a solution in $\left(\mathcal{B}, \mathcal{N}^{0}, \mathcal{N}^{1}\right)$. (The definition of a subgraph is straightforward.)

Take a ground node $a_{0} \in V G_{1}$, where $G_{1}$ is some component, and let $G=$ $\bigcup_{j=1}^{K} G_{j}$ be a finite union of components, which is 1-connected. Since $\widehat{V G}^{\prime \prime}$ is compact and has no limit points, it is a finite set $A$. For each $y \in A$ take $U(y) \in$ $U^{*}(y)$, mutually disjoint. By the compactness of $\widehat{V G}^{\prime} \backslash \bigcup\{U(y): y \in A\}$, since this has no limit points, it is a finite set $B$. For each $y$ in $B$ take $U(y)$ in $U^{*}(y)$ such that the members of $\{U(y): y \in A \cup B\}$ are mutually disjoint. By the compactness of $\widehat{V G} \backslash \bigcup\{U(y): y \in A \cup B\}$, since it has no limit points, this too is a finite set $C$ of nodes. Let $U$ stand for the set $\{U(y): y \in A \cup B\}$ or the function taking $y \in A \cup B$ to $U(y)$. Accordingly, we write $A=A(U), B=B(U)$, and $C=C(U)$.

We form a finite digraph $\left(\mathcal{B}_{U}, \mathcal{N}_{U}\right)$ as follows. We take $\mathcal{N}_{U}=\widehat{V G} / S$, where $S$ is the equivalence relation on $\widehat{V G}$ given by $x S x$ for all $x$ and $x S y$ if there are terminals $x_{1}$ and $y_{1}$ in the same 1-node with $x \in U\left(x_{1}\right)$ and $y \in U\left(y_{1}\right)$. For $\mathcal{B}_{U}$ we take all branches in $E G$ incident to $C$ (each branch being incident through one of 
its ends but not necessarily both). We define an incidence relation $\left(\partial_{U}^{+}, \partial_{U}^{-}\right)$from $\mathcal{B}_{U}$ to $\mathcal{N}_{U}$ by $\partial_{U}^{+} b=S\left(\partial^{+} b\right)$ and $\partial_{U}^{-} b=S\left(\partial^{-} b\right)$.

We may assume that $\left(\mathcal{B}_{U}, \mathcal{N}_{U}\right)$ is connected by taking $U(y)$ small enough for each $y \in A \cup B$. We define a monotone network $\mathbf{M}_{U}$ on $\left(\mathcal{B}_{U}, \mathcal{N}_{U}\right)$ by taking $\left(M_{U}\right)_{b}=M_{b}$ for $b \in \mathcal{B}_{U}$.

By Minty's Theorem there is a solution $\left(i^{U}, v^{U}\right)$ of $\mathbf{M}_{U}$ with $\left|i_{b}^{U}\right| \leq \mathbb{I}$ and $\left|v_{b}^{U}\right| \leq \mathbb{V}$ for all $b \in \mathcal{B}_{U}$. Take $p^{U}$ as the potential for $v^{U}$ on $\mathcal{N}_{U}$ with $p^{U}\left(a_{0}\right)=0$. We define $P^{U}: \widehat{\mathcal{N}^{0}} \rightsquigarrow \mathbb{R}$ by $P^{U}(x)=p^{U}(S x)$ for $x \in \widehat{V G}$ and $P^{U}(x)=0$ for $x \in \widehat{\mathcal{N}^{0}} \backslash \widehat{V G}$.

Let $\mathcal{A}$ be the set of $U$ as above, i.e., for which there exists a subgraph $G=$ $G(U)=G_{1} \cup \ldots \cup G_{K}$ that is 1-connected, and, for each $y \in \widehat{V G}^{\prime \prime}, U(y) \in U^{*}(y)$, and, for all $y \in \widehat{V G}^{\prime} \backslash U\left(\widehat{V G}^{\prime \prime}\right), U(y) \in U^{*}(y)$, these all being disjoint, and any terminals in $G$ can be connected by a sequence in $\widehat{V G}^{\prime \prime} \cup\left(\widehat{V G}^{\prime} \backslash U\left(\widehat{V G}^{\prime \prime}\right)\right)$.

In order to obtain a solution $\left(i^{0}, v^{0}\right)$, we will use the Arzela-Ascoli Theorem [5]. For this purpose, we claim:

(a) for each $v \in \widehat{\mathcal{N}^{0}}$, the set $\left\{P^{U}(v): U \in \mathcal{A}\right\}$ is bounded, and

(b) the family $\left\{P^{U}: U \in \mathcal{A}\right\}$ is equicontinuous on $\widehat{\mathcal{N}^{0}}$.

We first establish (a). For $v \in \widehat{\mathcal{N}^{0}}$, by 1-connectedness there is a sequence $\left\{r_{1}, l_{2}, r_{2}, l_{3}, \ldots, l_{K}\right\}$ of terminals such that, for $j=2, \ldots, K-1, l_{j}$ and $r_{j}$ are terminals in a component $G_{j}, r_{1}$ being in the component $G_{1}$ with $a_{0} \in V G_{1}$ and $l_{K}$ being in the component $G_{K}$ with $v \in \widehat{V G_{K}}$. Here, $r_{j}$ and $l_{j+1}(j=1, \ldots, K-1)$ are in the same 1-node. Indeed, according to Corollary 2.6, we have an endless path $P_{j}$ connecting $l_{j}$ and $r_{j}$ for $2 \leq j \leq K-1$, with $P_{0}$ connecting $a_{0}$ and $r_{1}$ and $P_{K}$ connecting $l_{K}$ and $v$. Before bounding $\left|P^{U}\left(l_{j}\right)-P^{U}\left(r_{j}\right)\right|$, we bound $v_{b}^{U}$, where $b \in \mathcal{B}_{U}$. Now, $\left(i_{b}^{U}, v_{b}^{U}\right) \in M_{b}$, and $\left( \pm \delta_{b}^{\prime}\left(0,0, M_{b}\right), 0\right) \in M_{b}$. (Here, we use either the + or - sign according to whether the graph $M_{b}$ passes to the right or left of the origin.) Also, $R_{b}$ is a Lipschitz constant for $M_{b}$ on $[-\mathbb{I}, \mathbb{I}]$. Hence, $\left|v_{b}^{U}\right| \leq R_{b}\left|i_{b}^{U} \mp \delta^{\prime}\left(0,0, M_{b}\right)\right| \leq 2 \mathbb{I} R_{b}$. Thus, for $j=2, \ldots, K-1$,

$$
\left|P^{U}\left(l_{j}\right)-P^{U}\left(r_{j}\right)\right| \leq \sum_{b \in P_{j} \cap \mathcal{B}_{U}}\left|v_{b}^{U}\right| \leq 2 \mathbb{I} \sum_{b \in P_{j}, R_{b}<\infty} R_{b}+\sum_{b \in P_{j}, R_{b}=\infty} \mathbb{V} \leq(\mathbb{V} \vee 2 \mathbb{I}) \sum_{b \in P_{j}} s_{b}
$$

giving $\left|P^{U}\left(l_{j}\right)-P^{U}\left(r_{j}\right)\right| \leq(\mathbb{V} \vee 2 \mathbb{I}) d_{s}\left(l_{j}, r_{j}\right)$, and likewise for $j=1$ and $j=K$. Thus, we may write

$$
\left|P^{U}\left(a_{0}\right)-P^{U}(v)\right| \leq(\mathbb{V} \vee 2 \mathbb{I})\left(d_{s}\left(a_{0}, r_{1}\right)+\sum_{j=1}^{K-1} d_{s}\left(l_{j}, r_{j}\right)+d_{s}\left(l_{K}, v\right)\right) .
$$

This establishes (a).

For (b), we wish to show that $\left\{P^{U}: U \in \mathcal{A}\right\}$ is equicontinuous at any terminal $e$, i.e., given $\epsilon>0$, there is a $\delta>0$ such that, if $d_{s}(x, e)<\delta$, where $x, e \in \widehat{V H}$ for some component $H$, then, for all $U \in \mathcal{A}$, we have $\left|P^{U}(x)-P^{U}(e)\right|<\epsilon$. Take $\delta=\epsilon /(\mathbb{V} \vee 2 \mathbb{I})$, and, for $x \in \mathcal{N}^{0}$ with $d_{s}(x, e)<\delta$, take a path $P$ connecting $x$ and $e$ with $\sum_{b \in P} s_{b}<\delta$. Let $w$ be the first node of $P$ in $U(e)$; thus, $w S e$ and $P^{U}(e)=P^{U}(w)$. Then,

$$
\left|P^{U}(e)-P^{U}(x)\right|=\left|P^{U}(w)-P^{U}(x)\right| \leq \sum\left|v_{b}^{U}\right| \leq(\mathbb{V} \vee 2 \mathbb{I}) \sum_{b \in P} s_{b}<\epsilon,
$$

and likewise, for $x \in \widehat{\mathcal{N}}^{\prime}$, taking $P$ to be an endless path connecting $x$ and $e$. 
Extend $i^{U}: \mathcal{B}_{U} \rightsquigarrow \mathbb{R}$ to $\mathcal{B}$ by setting $i_{b}^{U}=0$ for $b \notin \mathcal{B}_{U}$, giving $i^{U} \in[-\mathbb{I}, \mathbb{I}]^{\mathcal{B}}$. By the Ascoli-Arzela Theorem, the set $\left\{P^{U}: U \in \mathcal{A}\right\}$ is relatively compact in the compact open topology on $\mathbb{R}^{\widehat{\mathcal{N}^{0}}}$. Now, $\mathcal{A}$ is a directed set, where $U<W$ means that $\mathcal{G}_{U} \subset \mathcal{G}_{W}$, and moreover $W(y) \subset U(y)$ for $y \in \widehat{V G}^{\prime \prime}$ or $y \in \widehat{V G}^{\prime} \backslash U\left(\widehat{V G}^{\prime \prime}\right)$. Hence, there is a continuous potential $P^{0}: \widehat{\mathcal{N}^{0}} \rightsquigarrow \mathbb{R}$ such that the corresponding net $P: \mathcal{A} \rightsquigarrow \mathbb{R}^{\mathcal{N}^{0}}$ accumulates at $P^{0}$. First countability [5, Theorem XII.5.2] and the existence of a countable cofinal subset of $\mathcal{A}$ gives a subsequence $\left\{P^{U_{n}}\right\}$ converging uniformly on $\widehat{V G}$ for each component $G$ of $\left(\mathcal{B}, \mathcal{N}^{0}\right)$ by [5, Theorem XII.7.1]. According to Tychonoff's Theorem, by taking another subsequence, we may assume that $\left\{i^{U_{n}}\right\}$ converges pointwise on $\mathcal{B}$ to $i^{0} \in[\mathbb{I}, \mathbb{I}]^{\mathcal{B}}$. Since the coboundary $v^{0}$ of $\left.P^{0}\right|_{\mathcal{N}^{0}}$ satisfies $v^{U_{n}} \mapsto v^{0}$ pointwise, $\left|v_{b}^{0}\right| \leq \mathbb{V}$ for all $b \in \mathcal{B}$. Since each $M_{b}$ is closed, $\left(i_{b}^{0}, v_{b}^{0}\right) \in M_{b}$ for each $b$.

Let $n^{1}=\left\{f_{1}, \ldots, f_{r}\right\}$ be a 1-node, where the $f_{j}$ are terminals. Let $U^{0} \in \mathcal{A}$ be chosen such that $n^{1} \subset A\left(U^{0}\right) \cup B\left(U^{0}\right)$, i.e., all the $f_{j}$ not in $\left.\widehat{V G\left(U^{0}\right.}\right)^{\prime \prime}$ are in $\left.\widehat{V G\left(U^{0}\right.}\right)^{\prime} \backslash \bigcup_{y \in A\left(U^{0}\right)} U^{0}(y)$. For $U \in \mathcal{A}$ with $U^{0}<U, P^{U}\left(f_{j}\right)=P^{U}\left(f_{i}\right)$ for $f_{j}$ and $f_{i}$ in $n^{1}$, and hence $P^{0}$ is constant on $n^{1}$. This gives $P^{0}$ defined and continuous on $\widehat{\mathcal{N}^{0}} / R$ in the quotient topology, where $e R f$ means $e$ and $f$ are in the same 1-node.

Now, we have only to show that $i^{0}$ satisfies Kirchhoff's current law. For $x \in \mathcal{N}^{0}$, since $i_{b}^{U_{n}} \rightarrow i_{b}^{0}$ for each branch $b$ incident to $x$ and since $i^{U_{n}}$ satisfies Kirchhoff's current law at $x, i^{0}$ does also, i.e.,

$$
\sum_{\partial^{+} b=x} i_{b}^{0}=\sum_{\partial^{-} b=x} i_{b}^{0}
$$

We claim that, for $z$, an isolated terminal, $I\left(i^{0}, z\right)$ exists. Take $\epsilon<d\left(z, \widehat{V G}^{\prime} \backslash\{z\}\right)$. Since, for $b \in\left\langle\mathcal{N}^{0} \backslash B(z, \epsilon), \mathcal{N}^{0} \cap B(z, \epsilon)\right\rangle$, we have $\left(0, \pm \delta_{b}^{\prime \prime}\left(0,0, M_{b}\right)\right) \in M_{b}$ (here, we use the + (resp. - ) sign if $M_{b}$ passes above (resp. below) the origin) and $\left(i_{b}^{0}, v_{b}^{0}\right) \in M_{b}$, it follows that

$$
\left|i_{b}^{0}\right| \leq G_{b}\left|v_{b}^{0} \mp \delta_{b}^{\prime \prime}\left(0,0, M_{b}\right)\right| \leq 2 \mathbb{V} G_{b} .
$$

Hence,

$$
\sum\left\{\left|i_{b}^{0}\right|: b \in\left\langle\mathcal{N}^{0} \backslash B(z, \epsilon), \mathcal{N}^{0} \cap B(z, \epsilon)\right\rangle\right\} \leq 2 \mathbb{V} G_{z, \epsilon}<\infty
$$

by hypothesis (iii). Hence, since $\sum\left\{i_{b}^{0} \epsilon_{b}: b \in\left\langle\mathcal{N}^{0} \backslash B(z, \epsilon), \mathcal{N}^{0} \cap B(z, \epsilon)\right\rangle\right\}$ converges absolutely, $I\left(i^{0}, B(z, \epsilon) \cap \mathcal{N}^{0}\right)$ exists. (Here, $\epsilon_{b}=+1$ (resp. $\epsilon_{b}=-1$ ) if $b$ is directed into (resp. out of) $B(z, \epsilon)$.)

By Proposition 3.12(a), taking $M^{0}$ to be $B(z, \epsilon)$, we see that $I\left(i^{0}, z\right)$ exists and equals $I\left(i^{0}, M \cap \mathcal{N}^{0}\right)$ for all $M \in U^{*}(z)$; moreover,

$$
\left|I\left(i^{0}, z\right)\right| \leq 2 \mathbb{V} G_{z, \epsilon} .
$$

We first show that Kirchhoff's current law holds for $i^{0}$ at a 1 -node $n^{1}$, whose elements are all isolated terminals, and that, for $y \in n^{1}$,

$$
\left|I\left(i^{0}, y\right)\right| \leq 2 \mathbb{V} \sum_{z \in n^{1} \backslash\{y\}} G_{z, \epsilon(z)} .
$$


The inequality (10) follows immediately from (9) and Kirchhoff's current law. We need to show that $\sum_{y \in n^{1}} I\left(i^{0}, B(y, \epsilon(y)) \cap \mathcal{N}^{0}\right)=0$. But, $i^{0}=\lim _{n \rightarrow \infty} i^{U_{n}}$ pointwise on $\left\langle\mathcal{N}^{0} \backslash B(y, \epsilon(y)), \mathcal{N}^{0} \cap B(y, \epsilon(y))\right\rangle$ for each $y \in n^{1}$, and $\left|i_{b}^{U_{n}}\right| \leq 2 \mathbb{V} G_{b}$ with

$$
\sum_{y \in n^{1}} \sum\left\{2 \mathbb{V} G_{b}: b \in\left\langle\mathcal{N}^{0} \backslash B(y, \epsilon(y)), \mathcal{N}^{0} \cap B(y, \epsilon(y))\right\rangle\right\}<\infty .
$$

Therefore, by dominated convergence,

$$
\begin{aligned}
\sum_{y \in n^{1}} I\left(i^{0}, B(y, \epsilon(y)) \cap \mathcal{N}^{0}\right) & =\lim _{n} \sum_{y \in n^{1}} I\left(i^{U_{n}}, B(y, \epsilon(y)) \cap \mathcal{N}^{0}\right) \\
& =\lim _{n} \sum_{y \in n^{1}} I\left(i^{U_{n}}, U_{n}(y) \cap \mathcal{N}^{0}\right) \quad(\text { by Proposition 3.12(a) }) \\
& =0 .
\end{aligned}
$$

We now claim that, for $y \in \widehat{\mathcal{N}}^{\prime \prime}$, the flow $I\left(i^{0}, y\right)$ of $i^{0}$ into $y$ exists. Take $0<\epsilon<d\left(y, \widehat{\mathcal{N}}^{\prime \prime} \backslash\{y\}\right)$ with $G_{y, \epsilon}(D)=\sum_{b \in D} G_{b}<\infty$ by hypothesis (iv). Then, $\sum_{b \in D}\left|i_{b}^{0}\right| \leq 2 \mathbb{V} G_{y, \epsilon}(D)<\infty$. Hence, $I\left(i^{0}, B(y, \epsilon) \cap \mathcal{N}^{0}\right)$ exists since $\sum\left\{i_{b}^{0} \epsilon_{b}\right.$ : $\left.b \in\left\langle\mathcal{N}^{0} \backslash B(y, \epsilon), \mathcal{N}^{0} \cap B(y, \epsilon)\right\rangle\right\}$ converges absolutely $\left(\epsilon_{b}= \pm 1\right.$ as before). By Proposition 3.12(b), taking $M_{0}$ to be $B(y, \epsilon)$, we have that $I\left(i^{0}, M \cap \mathcal{N}^{0}\right)$ exists for $m \in U^{*}(y)$ such that $M \cap \widehat{\mathcal{N}}^{\prime \prime}=\{y\}$.

Now, we want $\lim _{M \in U^{*}(y)} I\left(i^{0}, M \cap \mathcal{N}^{0}\right)$ to exist. By Proposition 3.12(c), this follows if $\sum I\left(i^{0}, z\right)$ converges absolutely, where the summation is over all isolated terminals $z$ in $B(y, \epsilon)$. But, by (10), for any such $z,\left|I\left(i^{0}, z\right)\right| \leq 2 \mathbb{V} \sum_{z \in n^{1} \backslash\{y\}} G_{z, \epsilon(z)}$, and so, by hypothesis (v), $\sum_{z \in B(y, \epsilon)}\left|I\left(i^{0}, z\right)\right|<\infty$, where again the summation is over all isolated terminals $z$ in $B(y, \epsilon)$. This proves our claim. In addition, by Proposition 3.12(c), for $B(y, \epsilon) \in U^{*}(y)$,

$$
I\left(i^{0}, y\right)=I\left(i^{0}, B(y, \epsilon) \cap \mathcal{N}^{0}\right)-\sum\left\{I\left(i^{0}, z\right): z \in B(y, \epsilon) \cap\left(\widehat{\mathcal{N}}^{\prime} \backslash\{y\}\right)\right\} .
$$

Next, we show that Kirchhoff's current law holds at a general 1-node $n^{1}=\left\{z_{j}\right.$ : $j \in J\} \cup\left\{z_{k}: k \in K\right\}$, where $z_{j}$ is not isolated for $j \in J$ but $z_{k}$ is isolated for $k \in K$. By local finiteness, $J$ and $K$ are finite sets. We have $I\left(i^{0}, z\right)$ existing for all $z \in n^{1}$, and we claim that $\sum_{j \in J \cup K} I\left(i^{0}, z_{j}\right)=0$. We need to show, knowing that the following limits exist, that

$$
\sum_{j \in J} \lim _{M \in U^{*}\left(z_{j}\right)} I\left(i^{0}, M \cap \mathcal{N}^{0}\right)+\sum_{k \in K} \lim _{M \in U^{*}\left(z_{k}\right)} I\left(i^{0}, M \cap \mathcal{N}^{0}\right)=0 .
$$

So, we need only show that

$$
\lim _{n \rightarrow \infty} \sum_{j \in J} I\left(i^{0}, U_{n}\left(z_{j}\right) \cap \mathcal{N}^{0}\right)+\lim _{n \rightarrow \infty} \sum_{k \in K} I\left(i^{0}, U_{n}\left(z_{k}\right) \cap \mathcal{N}^{0}\right)=0 .
$$

As in the proof of Kirchhoff's current law for a 1-node containing only isolated terminals, the second term is $\lim _{n \rightarrow \infty} \sum_{k \in K} I\left(i^{U_{n}}, U_{n}\left(z_{k}\right) \cap \mathcal{N}^{0}\right)$. Consider the first 
term. For $j \in J$, we claim that $I\left(i^{U_{n}}, U_{n}\left(z_{j}\right) \cap \mathcal{N}^{0}\right) \rightarrow I\left(i^{0}, U_{n}\left(z_{j}\right) \cap \mathcal{N}^{0}\right)$. Now,

$$
\begin{aligned}
I\left(i^{0}, U_{n}\left(z_{j}\right) \cap \mathcal{N}^{0}\right) & \\
= & I\left(i^{0}, B\left(z_{j}, \epsilon\right) \cap \mathcal{N}^{0}\right)-\sum\left\{I\left(i^{0}, y_{j}\right): y_{j} \in\left(B\left(z_{j}, \epsilon\right) \backslash U_{n}\left(z_{j}\right)\right) \cap{\widehat{\mathcal{N}^{0}}}^{\prime}\right\} \\
= & I\left(i^{0}, B\left(z_{j}, \epsilon\right) \cap \mathcal{N}^{0}\right)-\sum_{y \in B\left(z_{j}, \epsilon\right) \cap \widehat{\mathcal{N}^{0}} \backslash\left\{z_{j}\right\}} \chi_{\widehat{\mathcal{N}^{0}} \backslash U_{n}\left(z_{j}\right)}(y) I\left(i^{0}, y\right) .
\end{aligned}
$$

by Proposition 3.12. (Here $\chi$ denotes the characteristic function.) Similarly,

$$
\begin{aligned}
I\left(i^{U_{n}}, U_{n}\left(z_{j}\right) \cap \mathcal{N}^{0}\right)= & I\left(i^{U_{n}}, B\left(z_{j}, \epsilon\right) \cap \mathcal{N}^{0}\right) \\
& -\sum_{y \in B\left(z_{j}, \epsilon\right) \cap \widehat{\mathcal{N}}^{\prime} \backslash\left\{z_{j}\right\}} \chi_{\widehat{\mathcal{N}^{0}} \backslash U_{n}\left(z_{j}\right)}(y) I\left(i^{U_{n}}, y\right) .
\end{aligned}
$$

Now,

$$
I\left(i^{0}, B\left(z_{j}, \epsilon\right) \cap \mathcal{N}^{0}\right)=\lim _{n} I\left(i^{U_{n}}, B\left(z_{j}, \epsilon\right) \cap \mathcal{N}^{0}\right)
$$

by dominated convergence since $\left|i_{b}^{U_{n}}\right| \leq 2 \mathbb{V} G_{b}$ for $b \in D$ and $\left|i_{b}^{U_{n}}\right| \leq \mathbb{I}$ for $b \in$ $\left\langle\mathcal{N}^{0} \backslash B\left(z_{j}, \epsilon\right), \mathcal{N}^{0} \cap B\left(z_{j}, \epsilon\right)\right\rangle \backslash D$. To prove our claim we need only use dominated convergence to show that the sum in (13) converges to the sum in (12). For $y \in$ $B\left(z_{j}, \epsilon\right) \cap \widehat{\mathcal{N}}^{\prime} \backslash\left\{z_{j}\right\}$,

$$
I\left(i^{0}, y\right)=\lim _{n} \chi_{\widehat{\mathcal{N}^{0}} \backslash U_{n}\left(z_{j}\right)}(y) I\left(i^{U_{n}}, y\right) .
$$

This is dominated by $2 \mathbb{V} \sum_{z \in n^{1} \backslash\{y\}} G_{z, \epsilon(z)}$ by (10). By (v), this is summable over $z$. Hence, the sum in (13) converges to the sum in (12), and together with (14) this proves our claim. Thus, the first term in (11) is equal to

$$
\lim _{n} \sum_{j \in J} I\left(i^{U_{n}}, U_{n}\left(z_{j}\right) \cap \mathcal{N}^{0}\right) \text {. }
$$

Since

$$
\sum_{j \in J} I\left(i^{U_{n}}, U_{n}\left(z_{j}\right) \cap \mathcal{N}^{0}\right)+\sum_{k \in K} I\left(i^{U_{n}}, U_{n}\left(z_{k}\right) \cap \mathcal{N}^{0}\right)=0
$$

for large $n,(11)$ holds.

\section{REFERENCES}

[1] B.D. Calvert, Infinite nonlinear resistive networks, after Minty, Circuits, Systems, and Signal Processing, 15 (1996), 727-733. MR 97k:94093

[2] B.D. Calvert, Unicursal resistive networks, Circuits, Systems, and Signal Processing, 16 (1997), 307-324. MR 98f:94026

[3] D.I. Cartwright, P.M. Soardi, and W. Woess, Martin and end compactifications for non-locally finite graphs, Trans. Amer. Math. Soc., 338 (1993), 679-693. MR 93i:60096

[4] V. Dolezal, Monotone Operators and Applications in Control and Network Theory, Elsevier, New York, 1979. MR 82e:93050

[5] J. Dugundji, Topology, Allyn and Bacon, Boston, 1965. MR 33:1824

[6] H. Flanders, Infinite networks. I: Resistive networks, IEEE Trans. Circuit Theory, CT-18 (1971), 326-331. MR 43:1751

[7] H. Freudenthal, Über die Enden diskreter Räume und Gruppen, Comment. Math. Helv., 17 (1944), 1-38. MR 6:277b

[8] R. Halin, Über unendliche Wege in Graphen, Math. Ann., 157 (1964), 125-137. MR 30:578

[9] H.A. Jung, Connectivity in infinite graphs, in Studies in Pure Mathematics (L. Mirsky, Ed.), Academic Press, New York, 1971. MR 43:4708 
[10] D. König, Theorie der endlichen und unendlichen Graphen, Akademische Verlagsgesellschaft M. B. H., Leipzig, 1936.

[11] T. Lyons, A simple criterion for transience of a reversible Markov Chain, Annals of Prob., 11 (1983), 393-402. MR 84e:60102

[12] M. Iri, Network Flows, Transportation, and Scheduling. Theory and Applications, Academic Press, New York, 1969. MR 43:7197

[13] G. Minty, Monotone networks, Proc. Royal Soc. London, 257 (1960), 194-212. MR 22:10920

[14] N. Polat, Aspects topologiques de la séparation dans les graphes infinis. I, Math. Z., 165 (1979), 73-100. MR 80f:05040a

[15] P.M. Soardi, Approximation of currents in infinite nonlinear resistive networks, Circuits, Systems, and Signal Processing, 12 (1993), 603-612. MR 94d:94037

[16] P.M. Soardi, Potential Theory on Infinite Networks, Lecture Notes in Mathematics 1590, Springer Verlag, New York, 1994. MR 96i:31005

[17] W. Woess, Random walks on infinite graphs and groups - a survey on selected topics, Bull. London Math. Soc., 26 (1994), 1-60. MR 94i:60081

[18] A.H. Zemanian, Infinite Electrical Networks, Cambridge University Press, Cambridge, England, 1991. MR 93b:94034

[19] A.H. Zemanian, Connectedness in transfinite graphs and the existence and uniqueness of node voltages, Discrete Mathematics, 142 (1995), 247-269. MR 96d:05075

[20] A.H. Zemanian, Transfiniteness for Graphs, Electrical Networks, and Random Walks, Birkhauser, Boston, 1996. MR 97c:94027

Department of Mathematics, University of Aukland, Aukland, New Zealand

E-mail address: calvert@math.auckland.ac.nz

Electrical Engineering Department, SUny at Stony Brook, Stony Brook, New York $11794-2350$

E-mail address: zeman@ee.sunysb.edu 